\title{
Innate immunity in myasthenia gravis thymus: Pathogenic effects of Toll-like receptor 4 signaling on autoimmunity
}

\author{
Chiara Cordiglieri ${ }^{a}$, Roberta Marolda ${ }^{a}$, Sara Franzi ${ }^{a}$, Cristina Cappelletti ${ }^{a}$, \\ Carmelo Giardina $^{\mathrm{b}}$, Teresio Motta ${ }^{\mathrm{b}}$, Fulvio Baggi ${ }^{\mathrm{a}}$, Pia Bernasconi ${ }^{\mathrm{a}}$, Renato Mantegazza ${ }^{\mathrm{a}}$, \\ Paola Cavalcante ${ }^{\mathrm{a}, *}$ \\ a Neurology IV Unit, Neurological Institute 'Carlo Besta', Via Celoria 11, 20133 Milan, Italy \\ ${ }^{\mathrm{b}}$ Department of Pathological Anatomy, Azienda Ospedaliera Bolognini Seriate, Via Paterno 21, 24068 Seriate Bergamo, Italy
}

\section{A R T I C L E I N F O}

\section{Article history:}

Received 18 July 2013

Accepted 15 December 2013

\section{Keywords:}

Myasthenia gravis

Thymus

TLR4

Chemokines

Dendritic cells

Regulatory T-cells

\begin{abstract}
A B S T R A C T
The thymus is the main site of immune sensitization to $\mathrm{AChR}$ in myasthenia gravis (MG). In our previous studies we demonstrated that Toll-like receptor (TLR) 4 is over-expressed in MG thymuses, suggesting its involvement in altering the thymic microenvironment and favoring autosensitization and autoimmunity maintenance processes, via an effect on local chemokine/cytokine network. Here, we investigated whether TLR4 signaling may favor abnormal cell recruitment in MG thymus via CCL17 and CCL22, two chemokines known to dictate immune cell trafficking in inflamed organs by binding CCR4. We also investigated whether TLR4 activation may contribute to immunodysregulation, via the production of Th17-related cytokines, known to alter effector T cell (Teff)/regulatory T cell (Treg) balance. We found that CCL17, CCL22 and CCR4 were expressed at higher levels in MG compared to normal thymuses. The two chemokines were mainly detected around medullary Hassall's corpuscles (HCs), co-localizing with TLR4 ${ }^{+}$ thymic epithelial cells (TECs) and CCR4 ${ }^{+}$dendritic cells (DCs), that were present in higher number in MG thymuses compared to controls. TLR4 stimulation in MG TECs increased CCL17 and CCL22 expression and induced the production of Th17-related cytokines. Then, to study the effect of TLR4-stimulated TECS on immune cell interactions and Teff activation, we generated an in-vitro imaging model by co-culturing CD4 ${ }^{+}$Th1/Th17 AChR-specific T cells, naïve $\mathrm{CD} 4^{+} \mathrm{CD} 25^{+}$Tregs, DCs and TECs from Lewis rats. We observed that TLR4 stimulation led to a more pronounced Teff activatory status, suggesting that TLR4 signaling in MG thymic milieu may affect cell-to-cell interactions, favoring autoreactive T-cell activation. Altogether our findings suggest a role for TLR4 signaling in driving DC recruitment in MG thymus via CCL17 and CCL22, and in generating an inflammatory response that might compromise Treg function, favoring autoreactive T-cell pathogenic responses.
\end{abstract}

(c) 2013 Elsevier Ltd. All rights reserved.

\section{Introduction}

Myasthenia gravis (MG) is a T-cell dependent antibodymediated disease that affects neuromuscular junction (NMJ), causing weakness and fatigability of ocular and skeletal muscles. In

\footnotetext{
* Corresponding author. Neurology IV Unit - Neuromuscular Diseases and Neuroimmunology, Neurological Institute 'Carlo Besta', Via Celoria 11, 20133 Milan, Italy. Tel.: +3902 2394 4511/4651; fax: +39 0270633874

E-mail addresses: chiara.cordiglieri@istituto-besta.it (C. Cordiglieri), roberta. marolda@istituto-besta.it (R. Marolda),sara.franzi@istituto-besta.it (S. Franzi), cristina.cappelletti@istituto-besta.it (C. Cappelletti), anatomiapat.seriate@bolognini. bg.it (C. Giardina), teresio.motta@bolognini.bg.it (T. Motta), baggi@istituto-besta.it (F. Baggi), pbernasconi@istituto-besta.it (P. Bernasconi),rmantegazza@istituto-besta.it (R. Mantegazza), pcavalcante@istituto-besta.it (P. Cavalcante).
}

most cases ( $\sim 80 \%$ ), the autoimmune attack is caused by autoantibodies against the acetylcholine receptor (AChR) of the postsynaptic NMJ [1].

Several lines of evidence indicate that the thymus is the main site of the autosensitization process in AChR-positive MG (AChR$M G)$ [2]. Hyperplastic MG thymus is characterized by the presence of B cells either infiltrating the thymic medulla (diffused hyperplasia or thymitis) or organized into ectopic germinal centers (GCs) forming follicles (follicular hyperplasia) [3]. AChR-specific T cells and autoantibody-producing plasma cells have been found in hyperplastic MG thymus [3], suggesting an ongoing intra-thymic autoimmune response to AChR.

Accumulating data show that innate immunity activation via Toll-like receptors (TLRs) and chronic inflammation characterize MG thymus [4-6]. TLR4 signaling is activated by both Gram- 
negative bacteria and viral components [7], and is one of the TLR family members suspected to play a role in MG [4]. In our previous studies, we demonstrated TLR4 over-expression and the presence of poliovirus-infected $\mathrm{TLR}^{+}$macrophages in the thymus of MG patients [4,8], suggesting dysregulated TLR4mediated innate immune responses. Activation of the immune system and increased expression of AChR- $\alpha$ subunit in thymic epithelial cells (TECs) [5,6] are possible TLR-mediated inflammatory mechanisms leading to intra-thymic AChR autosensitization. Published data showed that regulatory T cells (Tregs) from MG thymuses are functionally defective [9] and therefore unable to control the T-cell-mediated autoimmune response to AChR. Whether dysregulated innate immune response, through TLR4 or other TLRs, contributes to self-tolerance disruption in MG thymus via an effect on Treg-mediated immunoregulation is not completely understood.

Chemokines and their receptors are key regulators of lymphocyte trafficking; they are able to recruit $\mathrm{T}$ cells and antigen presenting cells (APCs) into sites of inflammation and orchestrate APC, $\mathrm{T}-$, and B-cell encounters in these sites [10]. The interactions among these cells, as well as the balance among different T-cell subtypes (i.e. Th1, Th2, Th17 and Tregs), may determine the outcome of the immune reactions; in this regard, the molecular settings regulating immune cell migration and interaction is of great importance. The Thymus and Activation-Regulated Chemokine CCL17 (TARC) and the Macrophage-Derived Chemokine CCL22 (MDC) are known to dictate trafficking of Th cells, Tregs and dendritic cells (DCs) to inflamed areas [11]. CCL17 and CCL22 are mainly produced by DCs and can be up-regulated by several stimulating factors, such as lipopolysaccharide (LPS), the prototypical TLR4 agonist $[12,13]$. The receptor for CCL17 and CCL22 is the C-C chemokine receptor type 4 (CCR4) [14,15], a G protein-coupled receptor known to be expressed in functionally distinct subsets of T cells, including activated Th2 cells and Tregs, and DCs [16,17].

CCL22 and CCR4 are normally expressed in the thymus and participate in thymopoiesis by regulating the migration of maturing thymocytes through this organ [18]. However, their role in the intra-thymic MG pathogenesis has been never explored.

Aim of this study was to asses the pathogenic effects of TLR4 signaling in MG thymus, by investigating whether this signaling may lead to altered CCL17 and CCL22 expression, and to immunodysregulation, via the production of Th17-related cytokines, known to impair Treg function and favor autoimmunity [19]. The TLR4 effect on chemokine expression was also studied in thymic epithelial cell cultures derived from experimental autoimmune myasthenia gravis (EAMG) animals [20]. Finally, to better address the effect of the inflamed pathologic thymic milieu on the cellular interactions and functions, we set-up an in vitro model by coculturing rat DCs, TECs, Tregs and AChR-specific Th1/Th17 effector cells (Teffs).

Our overall findings suggest that TLR4 signaling may participate in the intra-thymic pathogenesis of MG, by promoting the recruitment of CCR4 ${ }^{+}$DCs in the inflamed MG thymus, where the ongoing TLR4-mediated innate immune responses may favor autoantigen presentation and alter Teff/Treg balance.

\section{Materials and methods}

\subsection{MG patients and thymuses}

The study was carried out in 12 thymuses with follicular hyperplasia (MG-FH), 8 thymuses with diffused hyperplasia (MG-DH), and 8 involuted thymuses obtained from early-onset (EOMG; $\leq 50$ years of age) AChR-positive MG patients (22 females and 6 males), who underwent thymectomy as therapeutic treatment. Fifteen patients were treated with corticosteroids before thymectomy, and 13 were untreated or treated only with cholinesterase inhibitors. Patients' clinical characteristics are summarized in Table 1. Nine non-pathologic thymuses from cardiopathic patients (mean age: $31.9 \pm 17.0$ ) were also examined as controls. Written informed consent was obtained from patients and controls for the use of thymus for research purposes. The study was approved by the Ethic Committee of the Neurological Institute 'Carlo Besta'. For each thymus, some fragments were fixed in $10 \%$ formalin and others were snap-frozen and stored at $-80^{\circ} \mathrm{C}$.

\subsection{Animals and EAMG induction}

Female Lewis rats (6-8 week old, $n=20$ ) were purchased from Charles Rivers Laboratories International, maintained and bred at the animal house of the Institute "Carlo Besta". Animal studies were approved by the Institute (codes: IMP-03-11 and IMP-04-11), and performed in accordance with the Principles of Laboratory Animal Care (European Communities Council Directive 86/609/EEC). Animals were sacrificed after deep anesthesia by exposure to carbon dioxide. EAMG was induced via immunization with purified Torpedo californica electroplax tissue (Aquatic Research Consultants) AChR (TAChR; $50 \mu \mathrm{g} / \mathrm{rat}$ ) in Complete Freund's Adjuvant (CFA; Beckton Dickinson-BD-Difco) or with the rat immunodominant AChR epitope, R97-116 (200 $\mu \mathrm{g} / \mathrm{rat})$, in CFA [21]. At chronic disease stage, 2 animals/ group were sacrificed to obtain thymic cell suspensions for primary TEC cultures. Control primary TEC cultures were obtained from ageand sex-matched PBS/CFA-immunized $(n=2)$ and naïve $(n=2)$ animals. EGFP ${ }^{+/+}$and EGFP ${ }^{+/-}$transgenic Lewis rats [22] were used for Tcell primary cell cultures and bone marrow-derived DCs.

\subsection{Immunohistochemistry (IHC) on thymic sections}

Six- $\mu \mathrm{m}$ thick serial sections from snap-frozen thymic tissues ( $n=4$ for each thymic pathology and control) were immunostained with primary antibodies specific for: CCL17 (Santa Cruz); CCL22 (Peprotech); CCR4 (BD Pharmingen); TLR4 (Santa Cruz); cytokeratin (CK; Dako); desmin (Dako); DC-LAMP (Immunotech); FoxP3 (Thermo Scientific) and CD20 (Dako); non-immune IgG staining was used as isotype control. Secondary labeling was performed with HRP Anti-Mouse or Anti-Rabbit antibodies (Dako) followed by incubation with 3,3' diaminobenzidine (DAB; Dako) and hematoxylin counterstaining. Images were digitally acquired with the ScanScope system (Aperio). DC-LAMP ${ }^{+}$cells were counted in normal $(n=3)$ and MG $(n=9)$ thymic sections and their number was normalized to medullary area size $\left(\mathrm{mm}^{2}\right)$.

Table 1

Clinical features of MG Patients included in the study.

\begin{tabular}{|c|c|c|c|}
\hline Thymic pathology & Follicular hyperplasia $(n=12)$ & Diffuse hyperplasia $(n=8)$ & Involuted thymus $(n=8)$ \\
\hline $\operatorname{Sex}(F: M)$ & $9: 3$ & $8: 0$ & $5: 3$ \\
\hline Early-onset ( $\leq 50$ years) & $12 / 12$ & $8 / 8$ & $8 / 8$ \\
\hline Age at surgery (years, mean $\pm S D$ ) & $28.6 \pm 8.4$ & $29.6 \pm 11.1$ & $28.5 \pm 10.9$ \\
\hline Ab AChR-positive & $12 / 12$ & $8 / 8$ & $8 / 8$ \\
\hline Immunosuppressive therapy & $5 / 12$ & $4 / 8$ & $6 / 8$ \\
\hline
\end{tabular}




\subsection{Double immunofluorescence (IF) on thymic sections}

Frozen $6-\mu \mathrm{m}$ thymic sections were immunostained with combinations of antibodies against CCL17, CCL22, CCR4 (BD Pharmingen or Abnova), peripheral-node addressing (PNAd; BD), CK, DC-LAMP, TLR4 (Santa Cruz) and FoxP3, followed by secondary labeling with Alexa Fluor 488-(Molecular Probes, Life Technologies), Cy3and Cy2-(Jackson Immunoresearch Lab.) conjugated goat antimouse and anti-rabbit IgGs, and $4^{\prime}, 6$-diamidino phenylindoledihydrochloride (DAPI; Life Technologies) staining. Images were captured via confocal laser-scanning microscopy (EZ-C1 scan-head equipped with Eclipse TE2000-E microscope; Nikon). Single- and double-positive cells were counted on 6 adjacent field areas per section at $\times 60$ magnification using Image J software v1.43u.

\subsection{Laser-capture microdissection (LCM)}

Normal ( $n=3)$ and MG $(n=9)$ thymuses were subjected to LCM of medullary areas containing Hassall's corpuscles (HCs) using a laser microdissector CellCut (MMI) equipped with an Eclipse
TE2000-S microscope (Nikon). For each thymus, three $15-\mu \mathrm{m}$ thick serial sections were mounted on the same LCM membrane slide, stained with hematoxylin and fixed in RNase-free 75\%-100\% ethanol. $500 \mu \mathrm{m}$-diameter medullary areas around each HC were selected, cut and pooled in a single cap and resuspended in lysis buffer. Sections devoid of microdissected areas were separately collected.

\subsection{Reverse transcription and real-time $P C R$}

Total RNA was extracted from frozen thymic fragments and cells using TRIzol reagent (Life Technologies), and from LCM samples with RNeasy Micro kit (Qiagen). DNase I-treated RNA was retrotranscribed using Superscript VILO cDNA synthesis kit or SSIII reagent (Life Technologies) and amplified in duplicates using predesigned functionally tested assays (Life Technologies). Human GAPDH and rat $\beta$-actin were used as endogenous controls (Life Technologies). GAPDH was stably expressed in thymic $(\mathrm{Ct}$ mean \pm SD: $23.25 \pm 0.49$ for normal thymuses; $22.85 \pm 0.48$ for MG thymuses) and TEC samples (Ct mean \pm SD: $19.91 \pm 0.48)$. $\beta$-actin
A

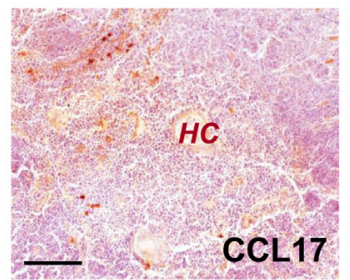

B

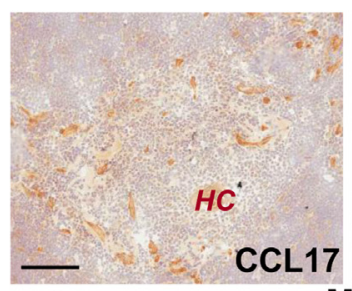

MG - Follicular hyperplasia

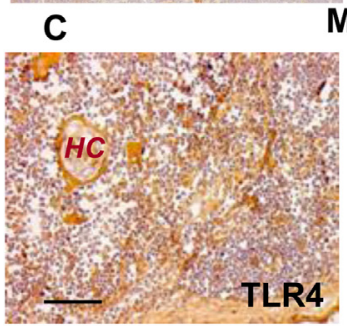

MG - Follicular hyperplasia
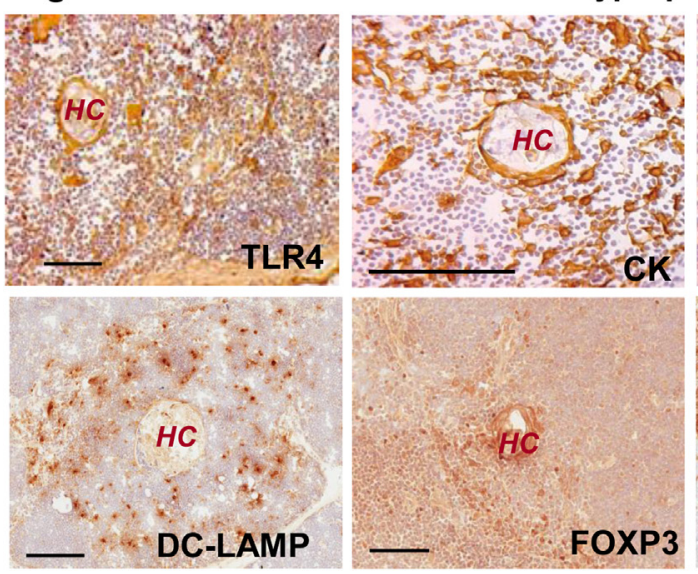

Normal thymus
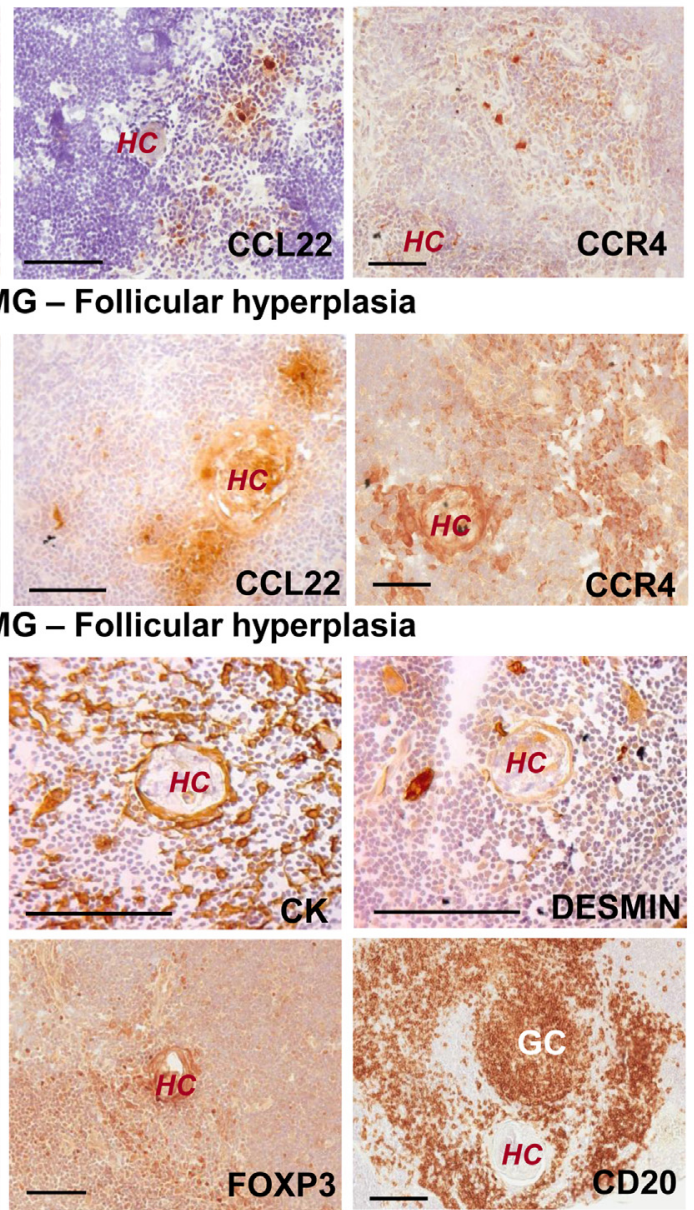

D MG - Follicular hyperplasia
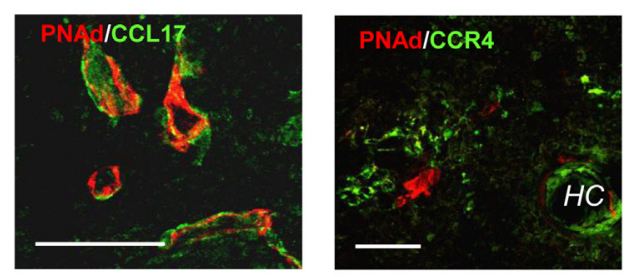

E
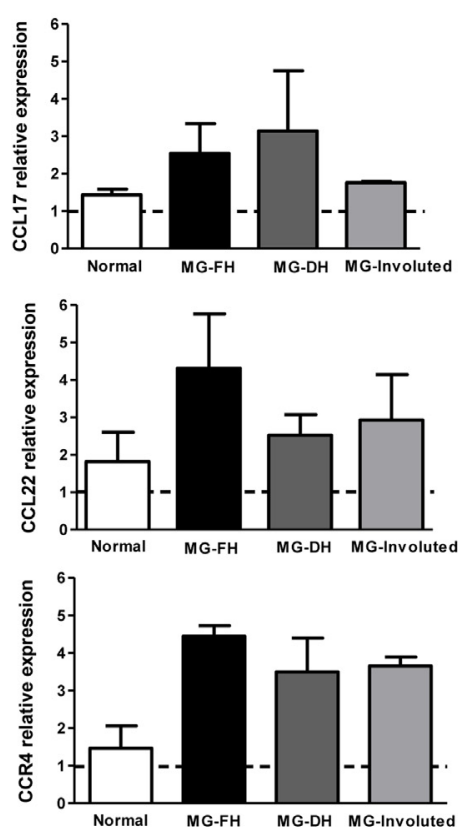

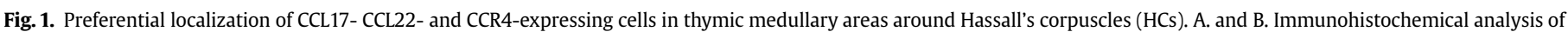

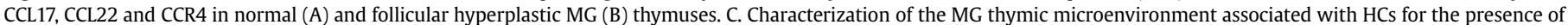

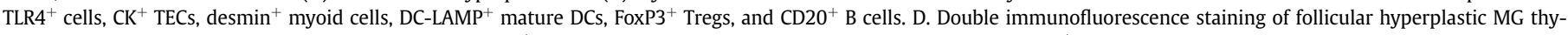

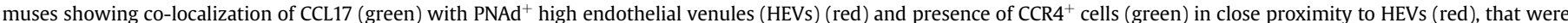

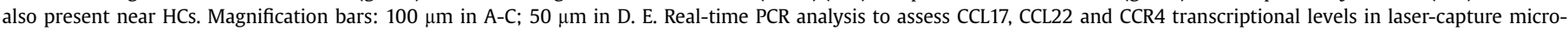

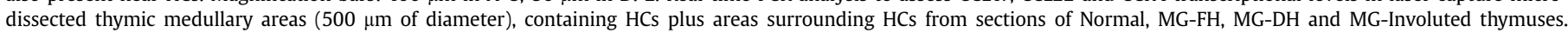

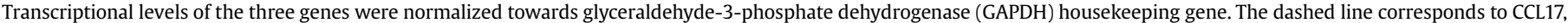

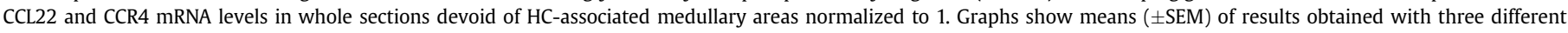
donors per group. 
was stably expressed in rat TECs (Ct mean \pm SD: $19.22 \pm 0.23)$ and co-cultures (Ct mean $\pm \mathrm{SD}$ : $17.67 \pm 0.35)$.

\subsection{Human and rat TEC isolation and TLR4 stimulation in vitro}

Human TEC primary cultures were established from freshly isolated hyperplastic thymuses of MG patients treated with cholinesterase inhibitors $(n=2)$ or corticosteroids $(n=2)$ prior to thymectomy. Finely cut thymic fragments were seeded into $60 \mathrm{~mm}$ collagen-coated dishes (BD) in culture medium (MEM with Dvaline, Cambrex Bioscience) supplemented with 10\% FBS, 2 mM Lglutamine, $100 \mathrm{U} / \mathrm{ml}$ penicillin, $0.1 \mathrm{mg} / \mathrm{ml}$ streptomycin, $10 \mathrm{mM}$ Hepes (all from Euroclone) and $10 \mathrm{ng} / \mathrm{ml}$ epidermal growth factor (EGF; Life Technologies). After 10-15 day culture, the confluent monolayers were detached and subcultured. Rat primary TECs were established from thymic tissues of naïve Lewis rats $(n=2)$, PBS/CFA- $(n=2)$, R97-116/CFA- $(n=2)$ or TAChR/CFA-immunized $(n=2)$ Lewis rats. Cell suspensions were cultured for 10-15 days into collagen type IV (Sigma)-coated $75 \mathrm{~mm}^{2}$ flasks in RPMI complete medium (10\% FBS, 2 mM L-glutamine, $100 \mathrm{U} / \mathrm{ml}$ penicillin, $0.1 \mathrm{mg} / \mathrm{ml}$ streptomycin) plus $10 \mathrm{ng} / \mathrm{ml}$ EGF (Peprotech). TEC purity ( $>85 \%$ ) was checked by anti-CK (Novocastra) IF staining followed by Alexa-488- or Cy2-conjugated IgGs. For TLR4 stimulation experiments, TECs $\left(1 \times 10^{5}\right.$ cells $\left./ \mathrm{cm}^{2}\right)$ were seeded in $5 \%$ FBScontaining culture medium for $24 \mathrm{~h}$ and treated with $1 \mu \mathrm{g} / \mathrm{ml}$ LPS (Sigma) for 3-48 h. In some experiments, human TECs were stimulated with LPS in the presence of $20 \mu \mathrm{g} / \mathrm{ml}$ of a cyanobacterial LPS antagonist (CyP) [23]. For IF stainings, $5 \times 10^{3}$ cells/well were plated on 4-well culture chamber slides (Nunc, Thermo Scientific), stimulated with LPS and immunostained with anti-TLR4 (Abcam) and anti-CK antibodies followed by Alexa-594 and -488 conjugated IgGs (Molecular Probes) and DAPI nuclei labeling. Images were acquired via confocal microscopy (Nikon).

\subsection{Analysis of human TEC protein secretion}

Supernatants were collected from human TECs at basal condition and after 24 and $48 \mathrm{~h}$ of LPS stimulation. Fifteen Th17-related proteins were measured by the Bio-Plex Pro Human Th17 Cytokine Assay kit (Bio-Rad Laboratories), following manufacturer's instructions.

\subsection{R97-116 specific effector rat Teff cell lines}

$\mathrm{EGFP}^{+/+}$transgenic and wild type (wt) Lewis rats were immunized with $200 \mu \mathrm{g}$ of peptide R97-116 in CFA as previously described [21]. Ten days post-immunization (p.i.) lymph node (LN) cell suspensions were cultured in RPMI medium, containing $1 \% \mathrm{Na}-$ pyruvate, $1 \%$ non-essential aa, $1 \%$ L-glutamine, $1 \%$ penicillinstreptomycin (Euroclone), $50 \mu \mathrm{M}$ 2-mercaptoethanol (Sigma), 2\% rat serum and stimulated with R97-116 (5 $\mu \mathrm{g} / \mathrm{ml})$ as described [24]. $\mathrm{T}$ cells were maintained and expanded as reported [25]. For live imaging, EGFP ${ }^{+/+}$R97-116-specific T cells were loaded with CFSE and wt R97-116-specific T cells were loaded with Fluo-4 dye to visualize intracellular calcium fluxes (both dyes from Molecular Probes).

\subsection{Rat DC cultures}

Single-cell suspensions of myeloid precursor cells (MPCs) were derived from bone marrows (BM) of naïve wt and $\mathrm{EGFP}^{+/-}$Lewis rats and cultured in complete RPMI medium in the presence of GMCSF and IL-4 (both $20 \mathrm{ng} / \mathrm{ml}$; Peprotech) for 10 days to differentiate into immature DCs (iDCs) [24]. iDCs were then seeded either in live imaging glass-inserted plates $\left(3 \times 10^{5} \mathrm{EGFP}^{+/-} \mathrm{DCs} / \mathrm{dish}\right)$ or transwell plates $\left(1 \times 10^{5}\right.$ wt DCs/well $)$ for specific assays.

\subsection{Rodent TEC/DC transwell assay}

Naïve wt TECs $\left(1 \times 10^{5}\right)$ were added in the lower chambers of 12-well transwell plates (Corning Costar, $3 \mu \mathrm{m}$ pore size) and incubated with LPS $(1 \mu \mathrm{g} / \mathrm{ml})$ for 12 and $24 \mathrm{~h}$. After medium change, differentiated wt iDCs were added in the upper chambers $\left(1 \times 10^{5}\right)$. After $24 \mathrm{~h}$ incubation, TECs and DCs were collected from bottom and lower chambers for RNA sampling and qPCR analysis.

\subsection{Rat Treg cultures}

Naïve natural occurring $\mathrm{CD} 127^{-} \mathrm{CD} 4^{+} \mathrm{CD} 25^{+}$rat Tregs were obtained from pool of LNs extracted from naïve wt Lewis rats. LN cell suspensions were processed using 3-step negative-positive MACS separation procedure (Miltenyi). Anti-CD127-Biotin, antiCD4-FITC and anti-CD25-PE antibodies (all eBioscience) and conjugate-specific magnetic beads (Miltenyi) were sequentially used. Tregs were finally labeled with CMRA dye (Molecular probes) and used in live imaging co-culture experiments $\left(8 \times 10^{5}\right.$ cells $/$ dish).

\subsection{Live imaging for Teff cell motility}

Time-lapse videomicroscopy was performed using a liveimaging Nikon set-up equipped with temperature $/ \mathrm{CO}_{2}$ control unit (OKOlab). Naïve wt TECs $\left(3 \times 10^{5}\right)$, $\mathrm{EGFP}^{+/-}$DCs $\left(3 \times 10^{5}\right)$, R97-116-specific CFSE-labeled EGFP ${ }^{+/+}$Teffs $\left(8 \times 10^{5}\right)$ and CMRAlabeled wt naïve Tregs $\left(8 \times 10^{5}\right)$ were co-cultured on glassinserted imaging collagen-coated dishes. Differential interference contrast (DIC), green and red channels images were acquired on a $512 \times 512$ pixel $^{2}$ field of view, with $1.31 \mu \mathrm{m} /$ pixel conversion. Recordings were performed in $30 \mathrm{~s}$ time intervals as previously reported [26] using an inverted microscope (with $20 \times, 0.5$ NA, objective) and a Q-imaging Fast Camera (Roper scientific), and processed by NIS Elements AR software v3.1 (Nikon). Image J software was used to evaluate cell trajectories and velocities. Cells were defined as stationary if they moved less than $10 \mu \mathrm{m} / 10 \mathrm{~min}$, motile if they moved more than $10 \mu \mathrm{m} /$ $10 \min [26,27]$.

\subsection{Cytosolic $\mathrm{Ca}^{2+}$ measurements of Teff cells}

Intracellular $\mathrm{Ca}^{2+}$ concentration $\left(\left[\mathrm{Ca}^{2+}\right]_{\mathrm{i}}\right)$ was determined in Fluo4-loaded R97-116-specific T cells $\left(8 \times 10^{5}\right.$ cells/dish) cocultured with DCs, TECs and CMRA-Tregs in the presence of appropriate stimuli (R97-116 $10 \mu \mathrm{g} / \mathrm{ml}$, LPS $1 \mu \mathrm{g} / \mathrm{ml}$ ) onto glassinserted imaging collagen-coated dishes. Calcium imaging was obtained via quick run-mode acquisition for $1 \mathrm{~h}$ using the liveimaging Nikon set-up previously cited. One DIC image was acquired at the beginning and at the end of each imaging for morphological cell analysis. Fluo-4 positive cells were counted and analyzed as percentage of total Teffs in each frame. $\left[\mathrm{Ca}^{2+}\right]_{i}$ calibration curve was performed using CD3/CD28 stimulation and ionomycin-induced calcium fluxes.

\subsection{Statistical analysis}

Data distribution was tested via Kolmogorov test: normally distributed data were analyzed via Student's $t$ test or one-/two-way ANOVA, followed by Bonferroni post-hoc test; non-parametric data were analyzed by Mann-Whitney $U$ test. $P<0.05$ was considered statistically significant. GraphPad Prism v5.0 (GraphPad Prism) was used for data elaboration and statistical analysis. 
A

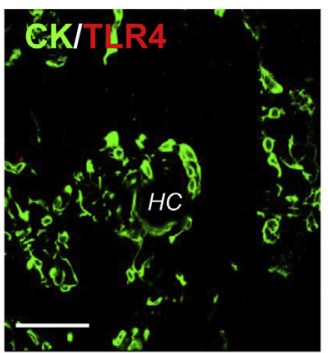

C
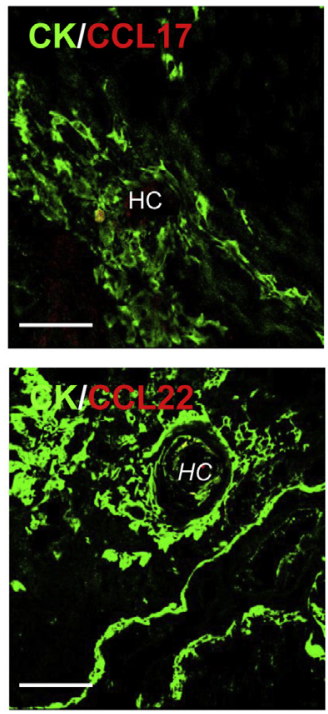

B

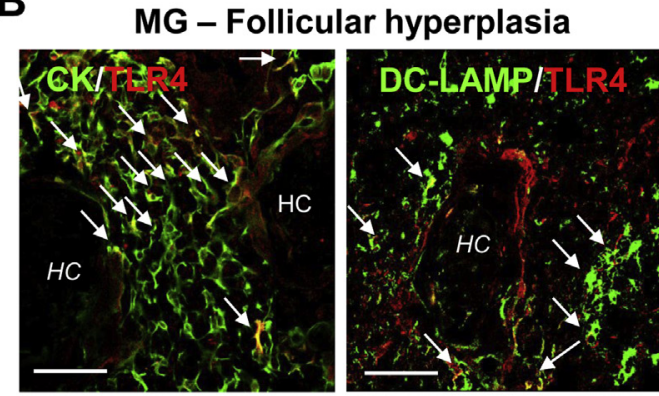

\section{D}
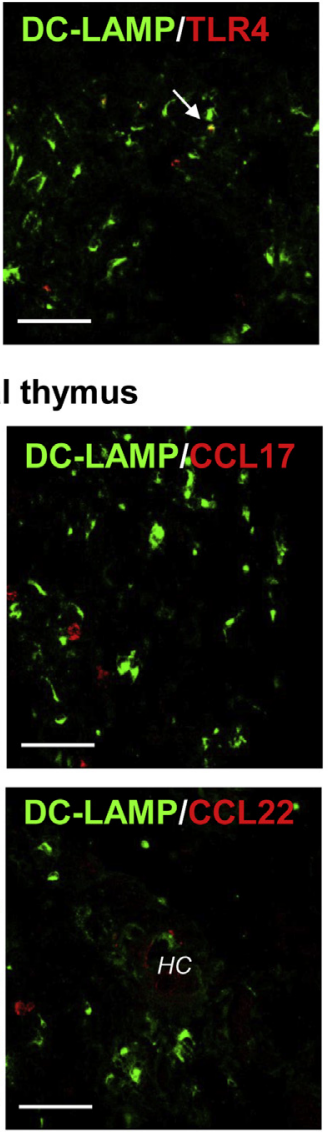

D
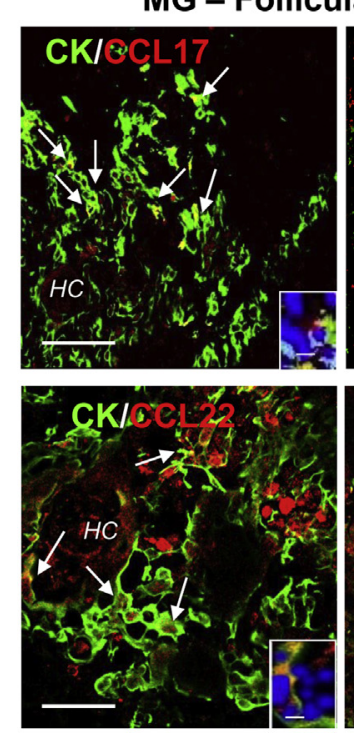

E

$$
\begin{aligned}
& \text { MG - Follicular } \\
& \text { hyperplasia }
\end{aligned}
$$
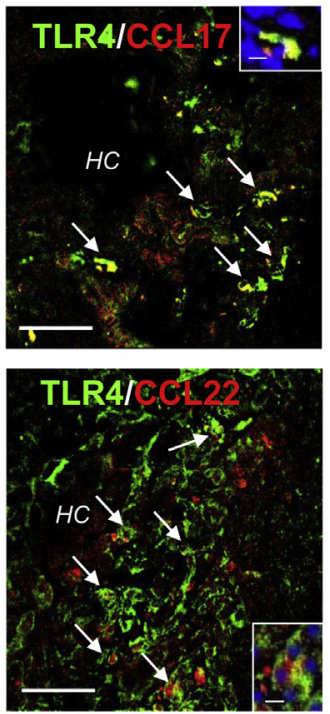

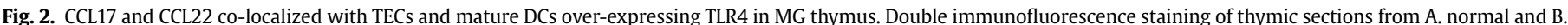

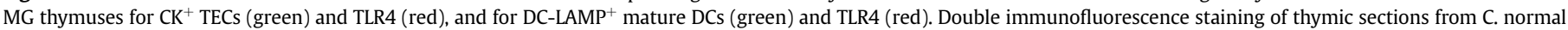

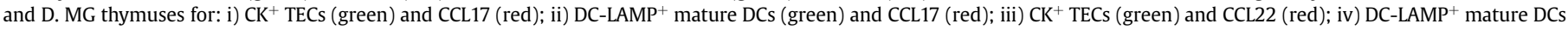

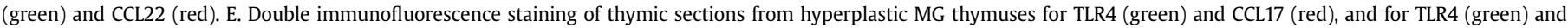

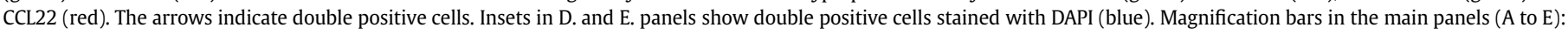
$50 \mu \mathrm{m}$; magnification bars in the insets (D and E): $10 \mu \mathrm{m}$. HC: Hassall's corpuscles.

\section{Results}

3.1. CCL17, CCL22 and CCR4 are enriched in medullary areas around HCs in MG thymus

Despite a similar distribution of $\mathrm{CCL}_{17}{ }^{+}$and $\mathrm{CCL} 22^{+}$cells in control and pathologic thymuses, being mainly observed in medullary areas containing HCs, an increased expression of CCL17 and CCL22 in HCs and in cells surrounding HCs was observed in MG thymuses (Fig. 1A and B; Supplementary Fig. 1). CCL17 was also expressed in PNAd ${ }^{+}$ HEVs, highly present in MG thymic medulla (Fig. 1D), suggesting that CCL17 might drive peripheral cell entrance via HEVs. An increased number of $\mathrm{CCR}_{4}^{+}$cells was found in MG thymuses, mainly concentrated around HCs, (Fig. 1A, B and D; Supplementary Fig. 1) and/or in close proximity to PNAd $^{+}$HEV (Fig. 1D).

CCL17- and CCL22-enriched thymic areas surrounding MG HCs showed a strong TLR4 positivity and presence of TECs, myoid cells, mature DCs, Tregs and B cells (Fig. 1C; Supplementary Fig. 1), suggesting that fine cellular interactions in these restricted medullary areas could play a role in thymic MG etiology.

Preferential CCL17, CCL22 and particularly CCR4 expression by HCs and surrounding cells was confirmed at mRNA levels on microdissected HC-associated areas, compared with whole thymic sections devoid of HCs (Fig. 1E).
3.2. CCL17 and CCL22 co-localize with TLR4 ${ }^{+}$TECS and mature DCS in MG thymus

To verify whether CCL17 and CCL22 expression in MG thymuses was linked to the expression of TLR4, we analyzed the two chemokines in TECs and DCs, known to over-express TLR4 in MG thymuses [4] (Fig. 2A and B). Indeed, CCL17 and CCL22 co-localized with $\mathrm{CK}^{+}$TECs and DC-LAMP ${ }^{+}$mature DCs more prominently in MG thymuses, and especially in microenvironment around HCs (Fig. 2C and D), and with TLR4 (Fig. 2E). This suggested an expression of the two chemokines by TLR4 ${ }^{+}$TECs and/or DCs in MG thymus.

\subsection{Increased number of CCR4-bearing DCs in MG thymus}

We wondered whether inflamed MG thymus was characterized by altered proportions of CCR4 ${ }^{+}$DCs and CCR $4^{+}$Tregs. We found an increased proportion of mature highly ramified DC-LAMP ${ }^{+} \mathrm{DCs}$, but not of FoxP3 ${ }^{+}$Tregs, in MG compared to normal thymuses (Fig. 3A, $\mathrm{B}, \mathrm{C}$ and $\mathrm{F}$ ). DC-LAMP ${ }^{+}$DC increase was not merely due to expansion of medullary area in MG thymuses (Fig. 3D). Interestingly, the percentage of DC-LAMP ${ }^{+}$DCs expressing CCR4, but not of CCR4 ${ }^{+}$ $\mathrm{FoxP}^{+}$Tregs, was higher in MG compared to normal thymuses (Fig. 3A, B, E and G). 


\subsection{CCL17, CCL22 and CCR4 mRNA expression is increased in MG} thymuses over-expressing TLR4

Besides confirming up-regulated levels of TLR4 mRNA [4], qPCR analysis showed that CCL17 and CCL22 mRNA levels were significantly increased in both follicular and diffuse hyperplastic MG thymuses compared with controls, whereas CCR4 mRNA was increased in follicular hyperplastic MG thymuses only (Fig. 4A). Immunosuppressive treatment does not seem to affect thymic TLR4, CCL22 and CCR4 expression since the mRNA levels did not differ between untreated and corticosteroid-treated patients (Fig. 4B), whereas CCL17 transcriptional levels were significantly reduced in thymuses from corticosteroid-treated MG patients, thus suggesting that immunosuppressive treatment could normalize CCL17 levels in MG thymuses (Fig. 4B).

\subsection{TLR4 stimulation by LPS increases CCL17/CCL22 expression and} Th17-related cytokine production in MG TECS

Since CCL17 and CCL22 were found to co-localize with TECs in MG thymuses, we wondered whether TLR4 signaling could modulate their expression. We first demonstrated that LPS stimulation increased TLR4 expression in MG TECs, and IL-6 as control for a proper TRL4 activation (Supplementary Fig. 2). Then, we found that LPS stimulation increased CCL17 and CCL22 mRNA levels in MG TECs, with a peak at $6 \mathrm{~h}$ of stimulation (Fig. 4C). This effect was blocked by the specific LPS-antagonist CyP [23] (Fig. 4C), thus confirming LPS binding to TLR4.

To better understand the pathogenic role of TLR4 signaling in MG TECs, we investigated whether LPS stimulation induced secretion of Th17-related cytokines. We analyzed TEC culture supernatants at basal condition and after LPS stimulation ( 24 and $48 \mathrm{~h}$ ). The data confirmed high levels of IL- 6 at basal and stimulated conditions (Table 2), accompanied by a significant increase of IL-1 $\beta$ production after $24 \mathrm{~h}$ of LPS treatment. Moreover, we observed a slight but significant increase of IL- 4 and IFN- $\gamma$ after 48 h of LPS treatment Despite IL-17 family cytokines were secreted at low levels by TECs, we found a significant increase of IL-17F in MG TECs after $48 \mathrm{~h}$ of LPS stimulation, suggesting that IL-17F could participate in the proinflammatory cytokine network that characterizes MG thymus.

\subsection{Th-17-related genes are up-regulated in MG thymus}

To provide insights into the possible involvement of Th17related cytokines in the thymic MG pathogenesis, we verified whether the expression of IL-6, IL-17A, IL-17F, IL17C and TGF- $\beta 1$

A

Normal thymus

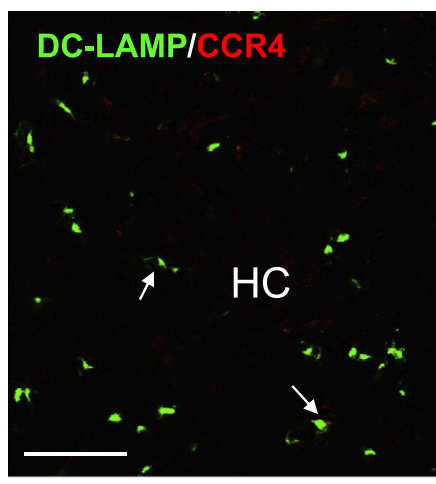

B

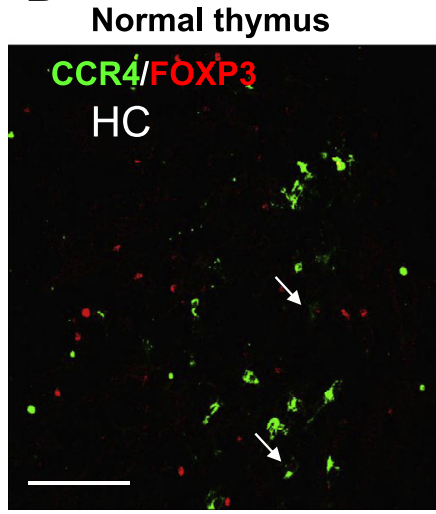

MG - Follicular hyperplasia

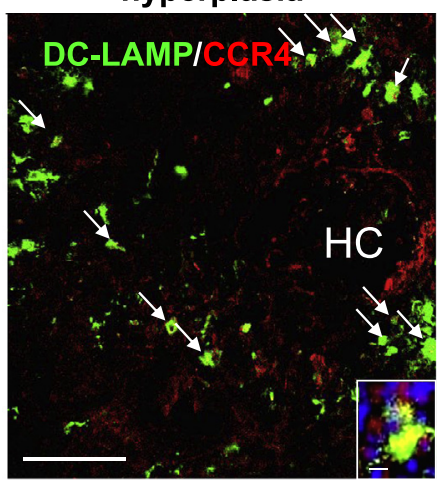

MG - Follicular hyperplasia

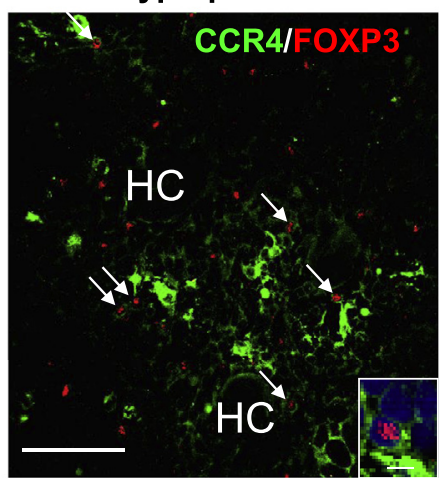

C

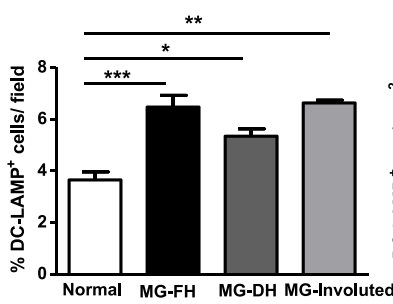

D
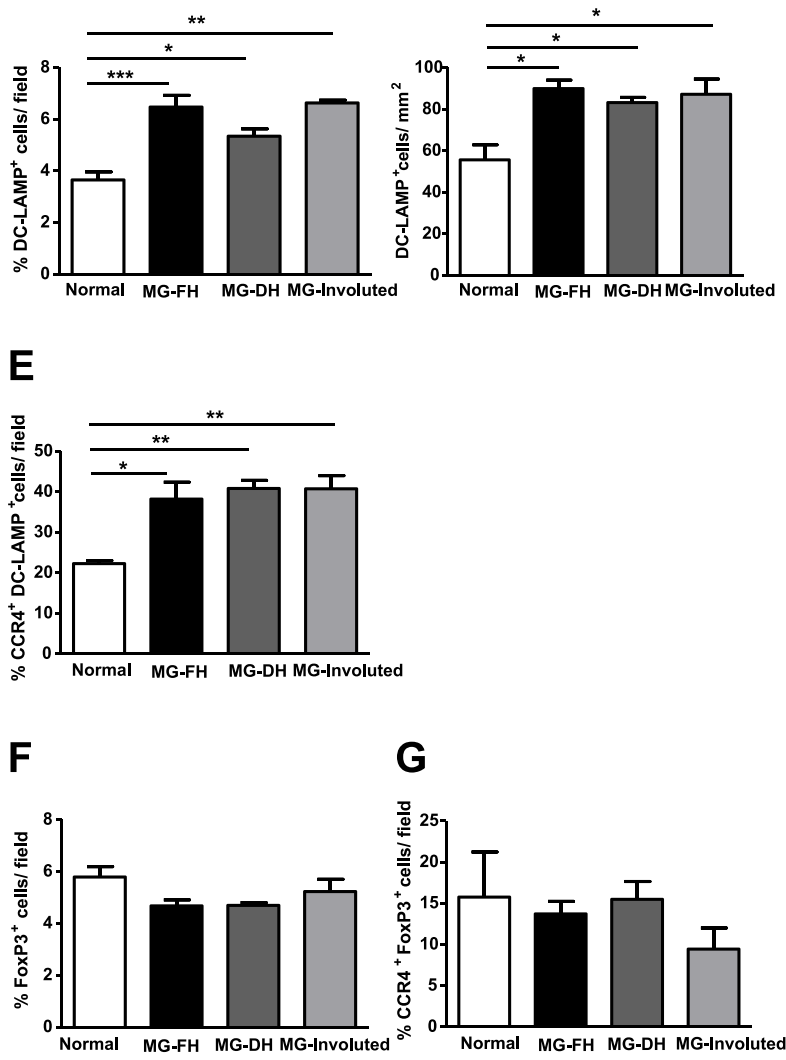

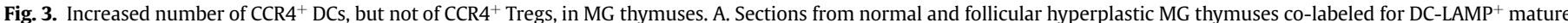

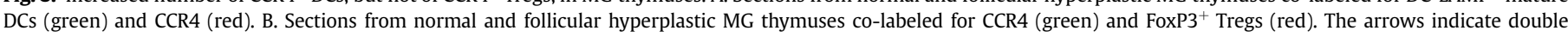

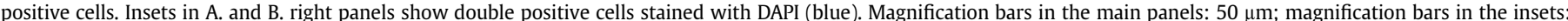

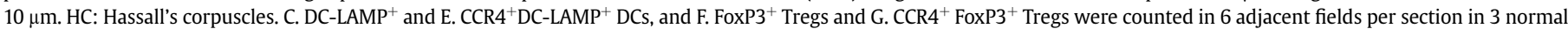

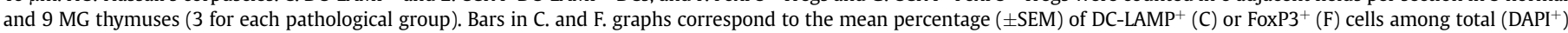

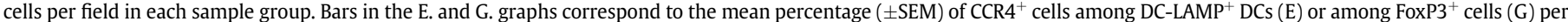

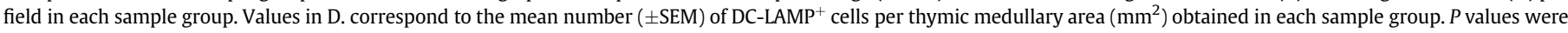
assessed by student $t$-test. ${ }^{*} P<0.05 ;{ }^{* *} P<0.01$; ${ }^{* * *} P<0.001$. 

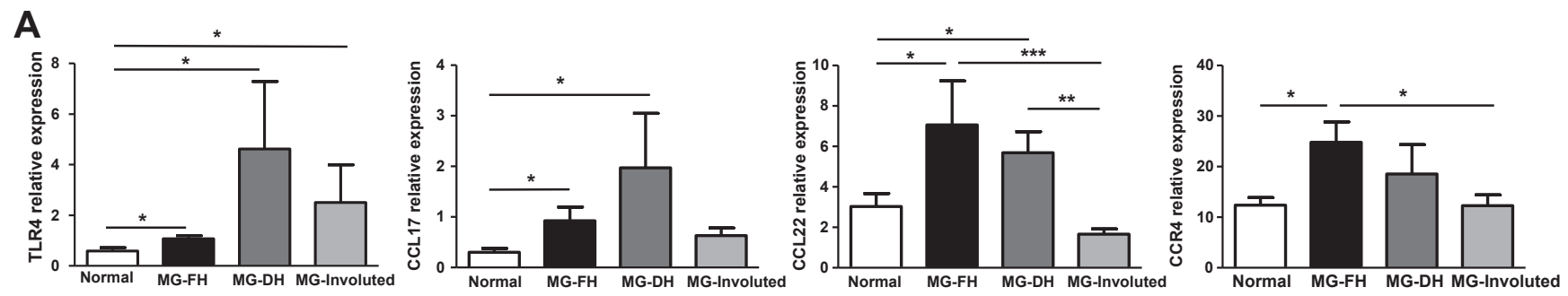

\section{B}
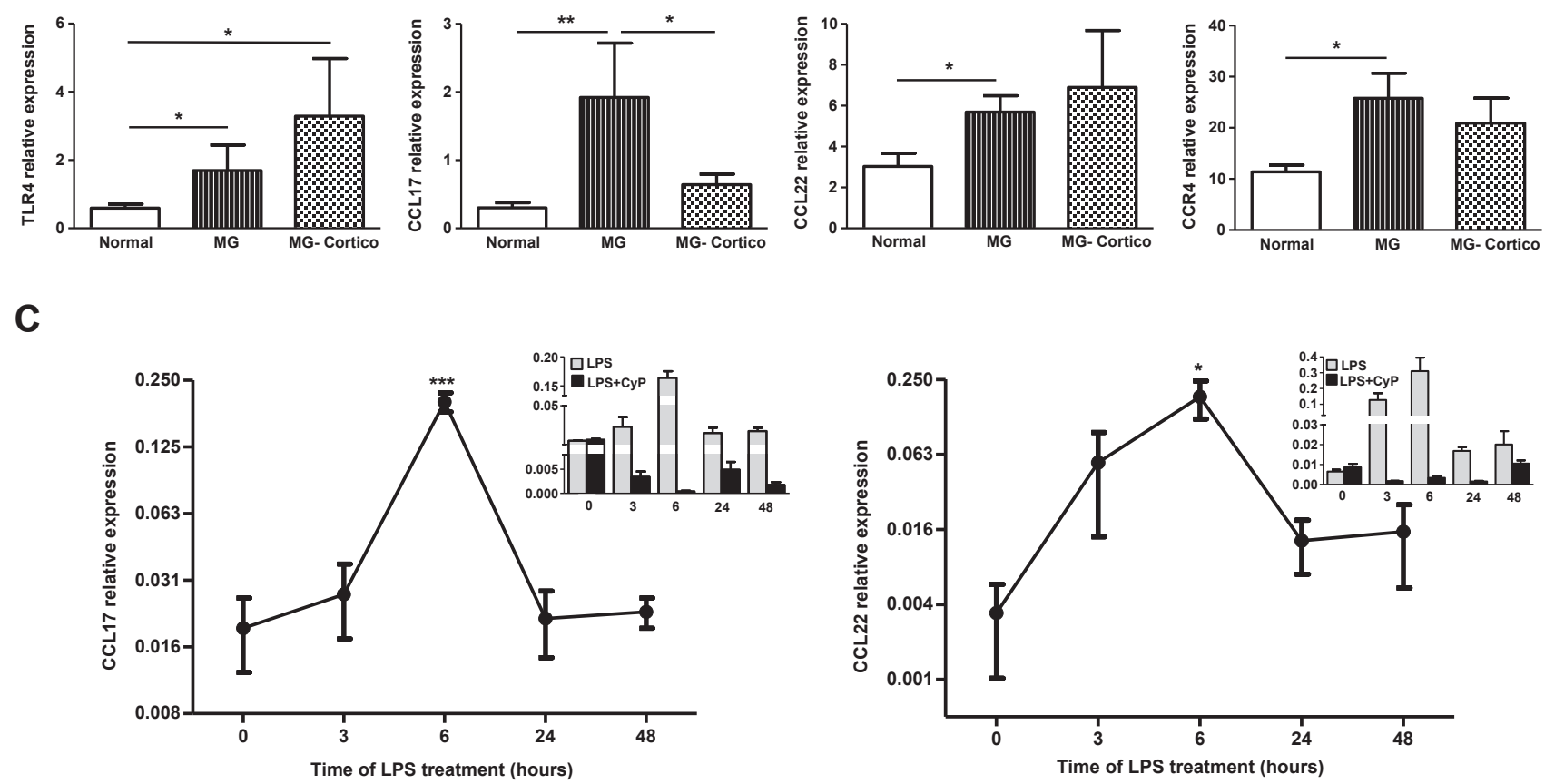

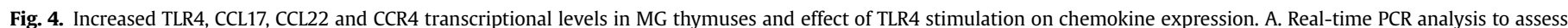

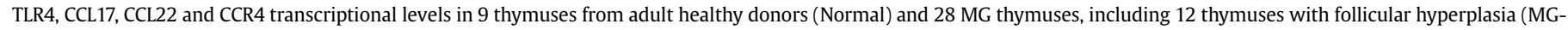

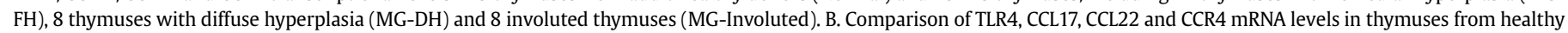

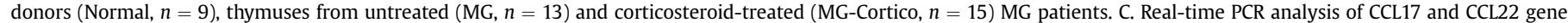

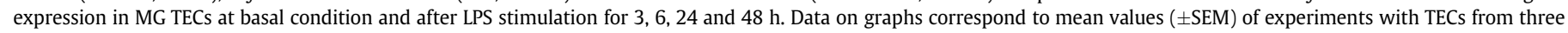

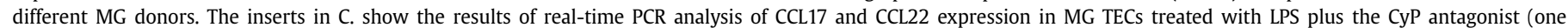

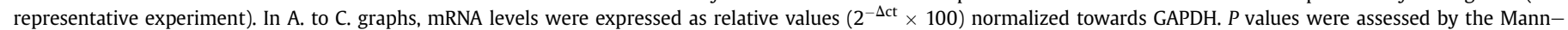
Whitney $U$ test $\left(\mathrm{A}\right.$ and $\mathrm{B}$ ) or by ANOVA followed by Bonferroni post-hoc test $(\mathrm{C})$. ${ }^{*} P<0.05 ;{ }^{* *} P<0.01 ;{ }^{* * *} P<0.001$.

was altered in MG thymuses. Increased IL-6, IL-17A and IL-17F, but not IL-17C, mRNA levels were found in MG thymuses compared with normal samples (Fig. 5): IL-6 gene expression was increased in each MG thymic pathology (Fig. 5A), whereas IL-17A and IL-17F were significantly increased in hyperplastic MG compared with normal thymuses (Fig. 5B and C), thus suggesting IL-17A and IL-17F involvement in MG thymic inflammatory state. The overexpression of these pro-inflammatory mediators was accompanied by a significant TGF- $\beta 1$ down-regulation in hyperplastic MG thymuses (Fig. 5E), and by a slight but not significant FoxP3 decrease (Fig. 5F). The expression of the investigated genes did not differ between untreated and corticosteroid-treated thymuses (Supplementary Fig. 3), except for IL-17F, which showed an indicative down-regulation in corticosteroid-treated thymuses (Supplementary Fig. 3C).

\subsection{TLR4 stimulation by LPS increases the expression of CCL17, CCL22 and CCR4 in rat EAMG TECS}

Similarly to human cells, TECs derived from EAMG, R97-116/CFA, PBS/CFA, and naïve Lewis rats showed an increase in TLR4 expression following LPS treatment, at mRNA and protein level (Fig. 6A and B), with a peak at $24 \mathrm{~h}$ post stimulation; whereas TLR4 basal expression was similar among the different TEC cultures, its LPS-induced increase was stronger in EAMG- and R97-116/CFAderived TECs. LPS stimulation of TEC cultures was accompanied by CCL17 and CCL22 up-regulation (Fig. 6A), which peaked after $6 \mathrm{~h}$ stimulation in naïve and PBS/CFA TECs, and after $12 \mathrm{~h}$ stimulation in EAMG- and R97-116/CFA TECs. Of note, CCR4 expression levels also significantly increased following LPS stimulation in both R97-116/ CFA and EAMG TECs, peaking after $12 \mathrm{~h}$ stimulation, compared with naïve and PBS/CFA TECs (Fig. 6A).

\subsection{TLR4 stimulation of rat TECs induces DC activation}

In order to study the effects of TLR4-stimulated TECs on DCs, we set-up a co-culture system with LPS-treated or untreated naïve rat TECs and DCs pre-pulsed with R97-116 peptide (Ag-DCs), at 1:1 cell ratio (Supplementary Fig. 4A). Ag-DCs cultured in the presence or absence of LPS were used as controls. A marked Ag-DC activation, visualized by morphological changes (Fig. 7A) and cell proliferation (Fig. 7B), was observed after $24 \mathrm{~h}$ of co-culture with LPS-stimulated 
Table 2

Effect of TLR4 stimulation by LPS on secretion of Th17-related cytokines in MG TECs.

\begin{tabular}{lccc}
\hline \multirow{2}{*}{ Gene symbol } & \multicolumn{3}{c}{ Time of LPS treatment } \\
\cline { 2 - 4 } & $0 \mathrm{~h}$ & $24 \mathrm{~h}$ & $48 \mathrm{~h}$ \\
\hline IL-1 $\beta$ & $152.40 \pm 31.64$ & $270.5 \pm 27.19^{*}$ & $216.20 \pm 56.84$ \\
IL-4 & $20.62 \pm 3.98$ & $25.30 \pm 4.90$ & $39.32 \pm 11.07^{* *}$ \\
IL-6 & $22210.00 \pm 871.86$ & $24128.57 \pm 124.39^{*}$ & $23932.00 \pm 478.00$ \\
IL-17F & $147.70 \pm 53.42$ & $109.6 \pm 54.28$ & $422.20 \pm 25.29^{*}$ \\
IFN- $\gamma$ & $29.98 \pm 5.98$ & $25.50 \pm 6.58$ & $72.87 \pm 8.07^{*}$ \\
IL-10 & $21.41 \pm 6.48$ & $18.46 \pm 2.67$ & $21.31 \pm 6.51$ \\
IL-17A & $50.35 \pm 34.76$ & $60.51 \pm 21.96$ & $39.50 \pm 17.53$ \\
IL-17C/IL-21 & $23.23 \pm 3.42$ & $26.27 \pm 7.32$ & $15.00 \pm 5.03$ \\
IL-22 & $187.30 \pm 69.71$ & $159.7 \pm 44.22$ & $115.40 \pm 25.73$ \\
IL-23 & $36.78 \pm 5.04$ & $26.39 \pm 16.36$ & $29.84 \pm 14.85$ \\
IL-17E/IL-25 & $17.77 \pm 3.93$ & $20.19 \pm 9.47$ & $13.03 \pm 4.10$ \\
IL-31 & $21.57 \pm 5.52$ & $18.04 \pm 9.75$ & $14.81 \pm 1.68$ \\
IL-33 & $21.54 \pm 7.58$ & $25.80 \pm 7.20$ & $19.83 \pm 1.50$ \\
SCD40L & $53.66 \pm 24.13$ & $42.37 \pm 26.39$ & $31.43 \pm 5.52$ \\
TNF- $\alpha$ & $23.07 \pm 1.08$ & $32.86 \pm 12.09$ & $19.79 \pm 4.11$ \\
\hline VIIIS & &
\end{tabular}

Values given in the table represent the mean concentration $(\mathrm{pg} / \mathrm{ml}) \pm \mathrm{SD}$ of each cytokine in supernatants of TECs from three different MG patients at basal condition $(0 \mathrm{~h})$ and after 24 and $48 \mathrm{~h}$ of LPS stimulation. Statistical differences between untreated and treated samples were assessed by ANOVA followed by Bonferroni post hoc tests. ${ }^{*} P<0.05,{ }^{* *} P<0.01$.

TECs and with direct LPS stimulation of Ag-DCs. Interestingly, untreated TECs induced a slight, but significant, Ag-DC activation (Fig. 7B). To evaluate whether Ag-DC activation was due to soluble factors from LPS-stimulated and untreated TECs, an indirect co- culture transwell set-up was established (Supplementary Fig. 4B). After $24 \mathrm{~h}$ of transwell co-culture, a significant increase in activation (CCR7, IL-6, IFN- $\gamma$ ) and immunomodulation (TGF- $\beta$ ) gene expression was observed when DC were exposed to soluble factors released from LPS-treated TECs (Fig. 7C). Untreated TEC medium induced down-regulation of Ag-DC endogenous IFN- $\gamma$ expression. Moreover, we observed that LPS-stimulated TECs induced a significant up-regulation of CCL17 and CCL22 expression in Ag-DCs, accompanied by TLR4 and CCR4 down-regulation (Fig. 7D), likely due to a post-activation response [28]. In contrast, direct Ag-DC LPS stimulation led to increased CCL17, CCL22, TLR4, and CCR4 increased expression, together with TGF- $\beta$ suppression (not shown), suggesting that the effect of LPS-activated TECs on Ag-DCs differs from direct LPS stimulation of Ag-DCs.

\subsection{Live-imaging of cellular interactions among TECs, DCs, Teff and Treg cells}

To address the cellular interactions that might occur in the inflamed thymic milieu, we performed in-vitro live imaging experiments, using multiple co-culture systems employing R97-116 specific Th1/Th17 effector cells (Teffs), naïve CD127 ${ }^{-} \mathrm{CD} 4^{+} \mathrm{CD} 25^{+}$ T cells (Tregs), Ag-DCs, and untreated or LPS-treated TECs, all from Lewis rats (Supplementary Fig. 4C). When co-cultured with Ag-DCs, R97-116 Teffs showed a random movement [29,30] (Fig. 8A; Supplementary Video 1), and then acquired a stationary phenotype when encountering the proper R97-116-MHC complex, showing a
A

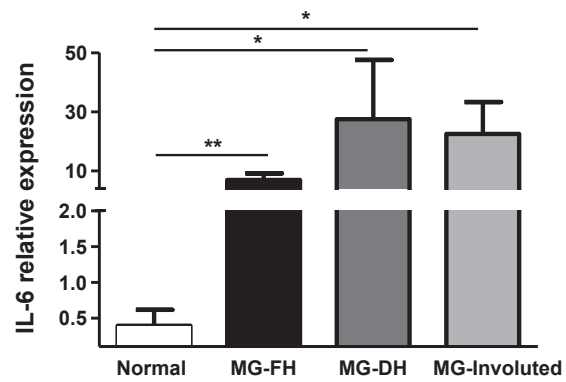

B

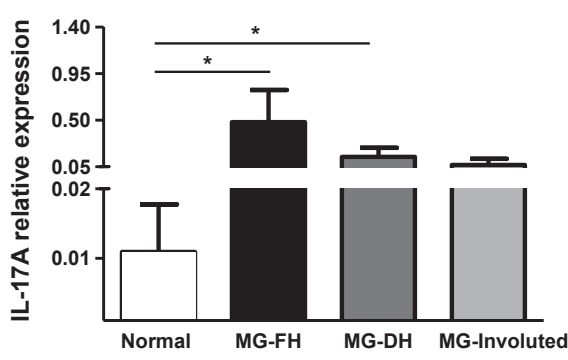

C

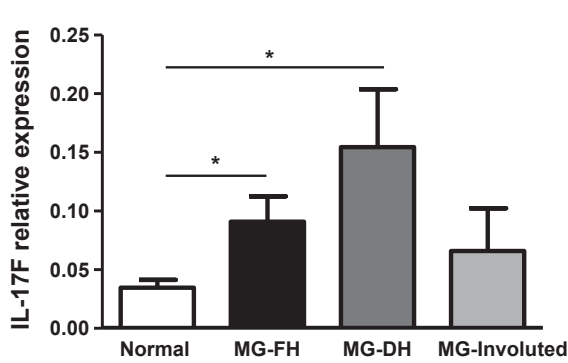

D

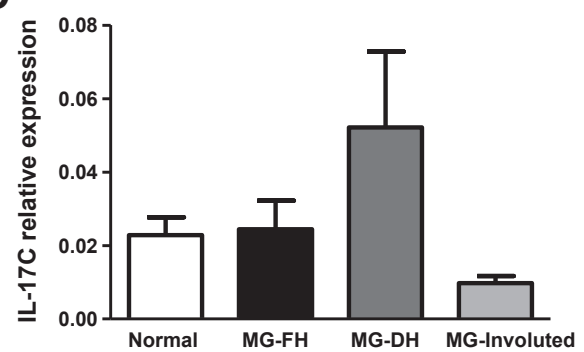

E

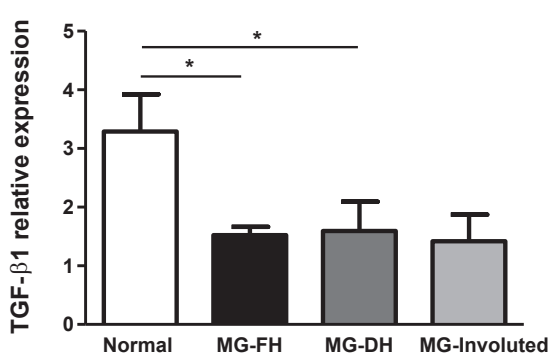

$\mathbf{F}$

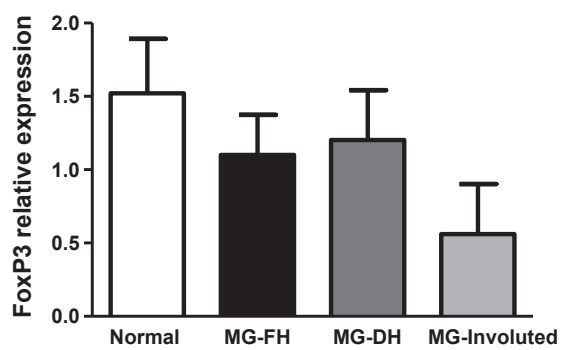

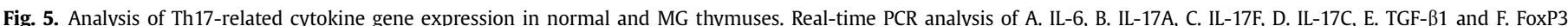

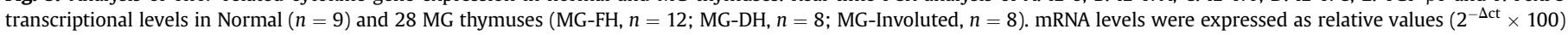

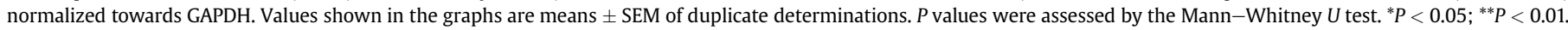



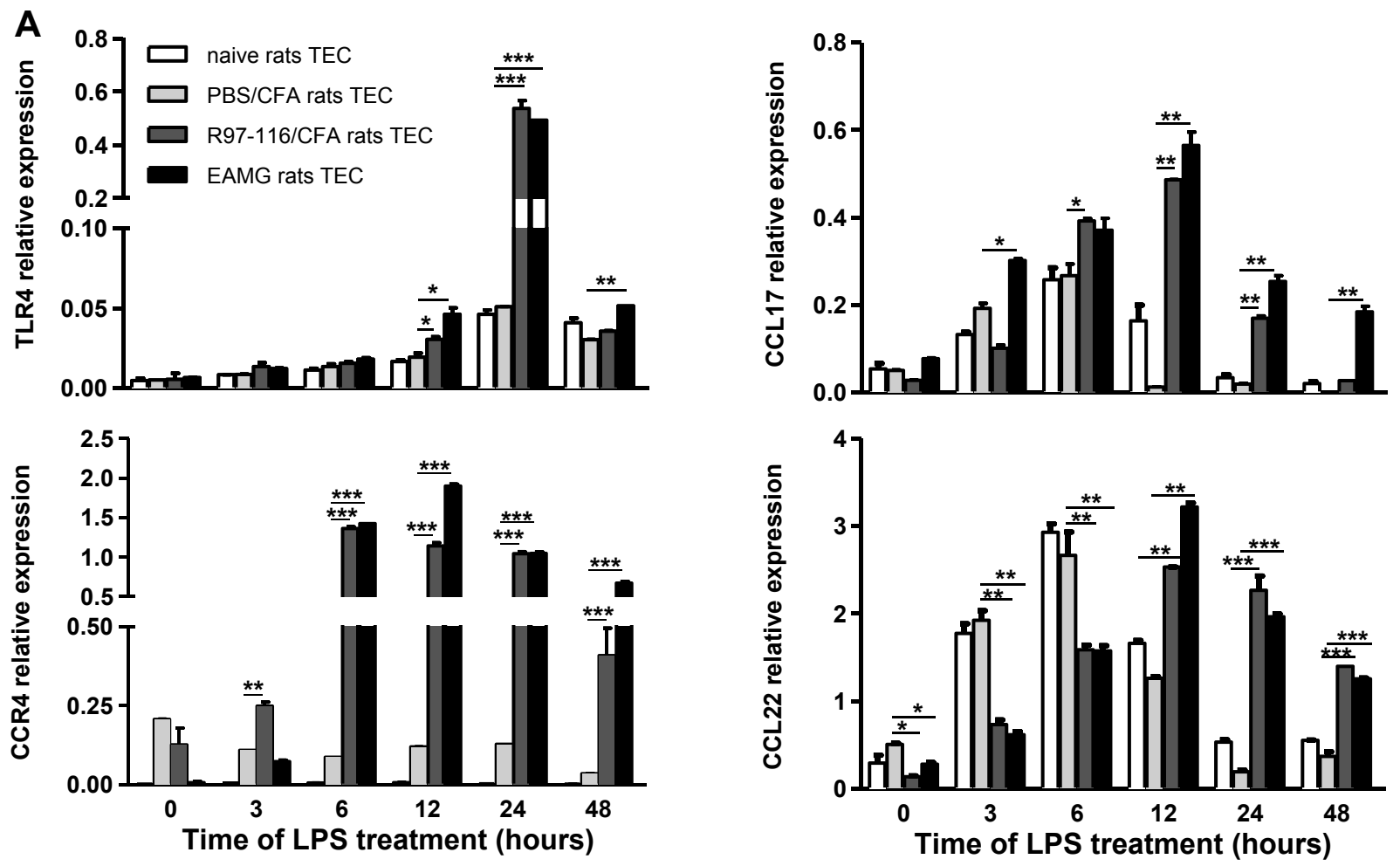

B

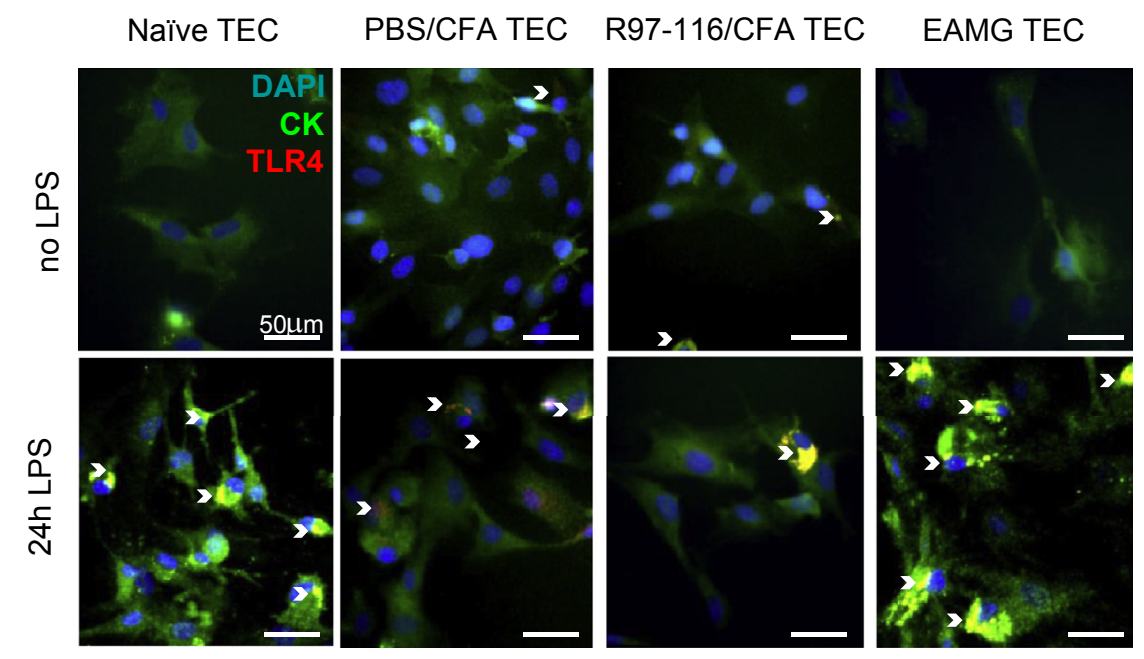

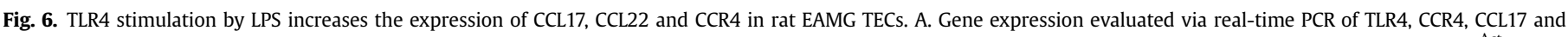

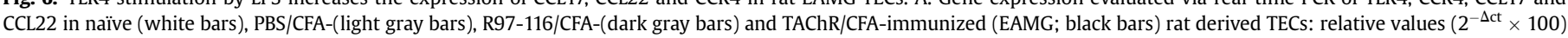

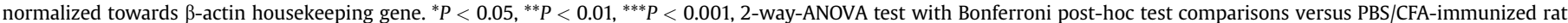

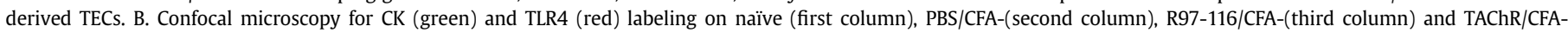

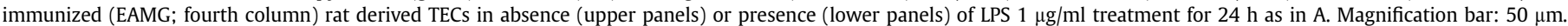
White arrowheads point to TLR4 ${ }^{+}$cells.

relatively stable low $V_{\max }(4 \mu \mathrm{m} / \mathrm{min}$ ), and remaining fixed to anchor points [26]. After 60 min co-culture, $65 \%$ of Teffs showed a stationary phenotype with a minimum meandering index (Table 3 ). Teff/Ag-DC contacts were long lasting, visually resembling immunological synapse formation (Fig. 8A, bottom panel; Supplementary Video 1).

When Tregs were added to Teffs/Ag-DCs co-culture (Fig. 8B; Supplementary Video 2), an increased Teffs $V_{\max }$ was observed at 60 -min co-culture (Table 3 ), and only $36 \%$ of Teffs presented a stationary phenotype; $52 \%$ of Teffs maintained a motile phenotype even after 90-min co-culture. Thus, Tregs interfered in Teff/Ag-DC stable contact formation, as confirmed by Teff high meandering index (Table 3; Supplementary Fig. 5). Treg/DC and Treg/Teff short (0.5-2 min) and medium (3-7 min) length contacts were visible throughout the imaging period, suggesting a competitor role for Tregs in forming contacts with both Teffs and DCs (Fig. 8B, bottom panel; Supplementary Video 2), as elsewhere hypothesized [31,32].

A similar motility pattern was observed when untreated TECs were added to Teffs/Ag-DCs/Tregs co-culture (Fig. 8C; Supplementary Video 3): high Teff $V_{\max }$ and low stationary Teff percentage (38\%) were measured at 60 -min co-culture (Table 3); $55 \%$ of Teffs maintained a motile phenotype till 90-min co-culture, and 
A

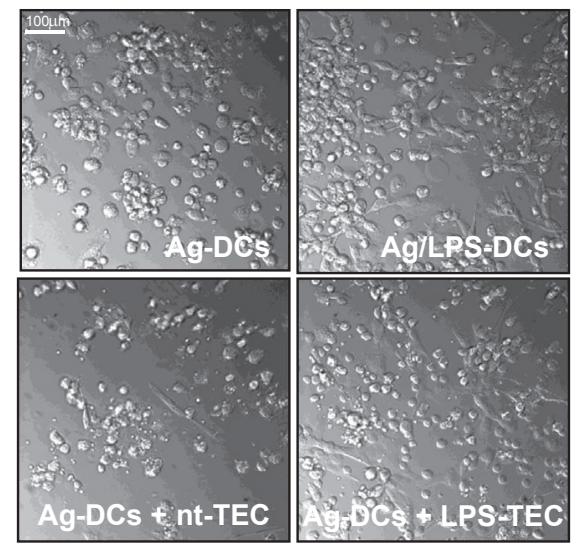

C

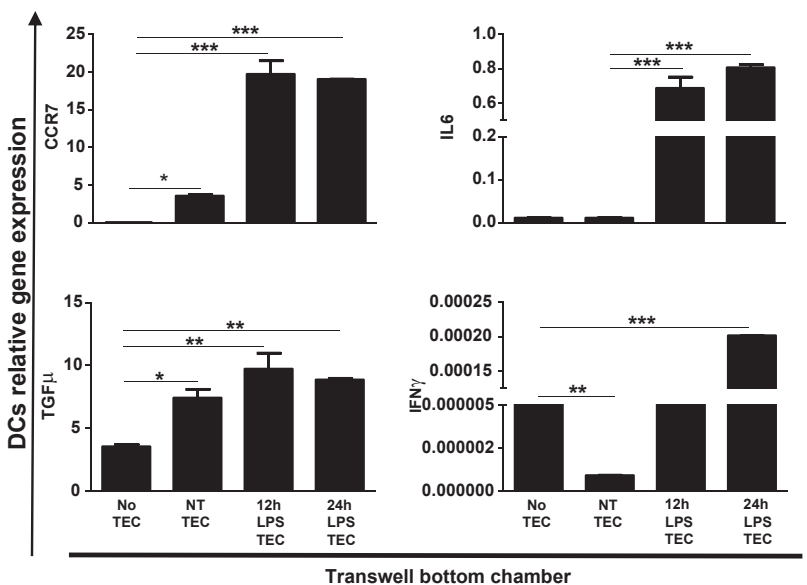

B

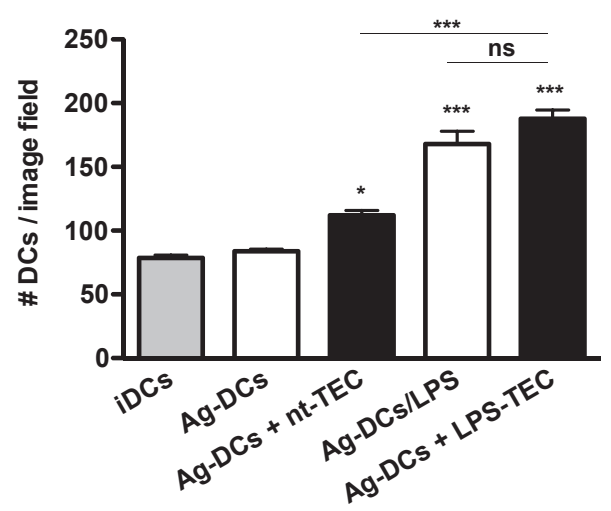

D

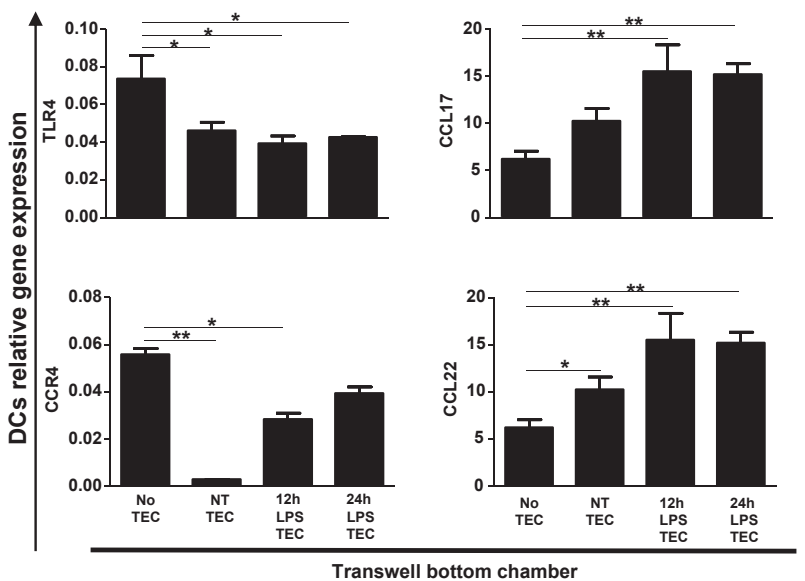

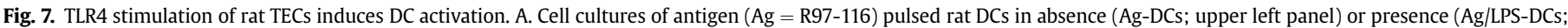

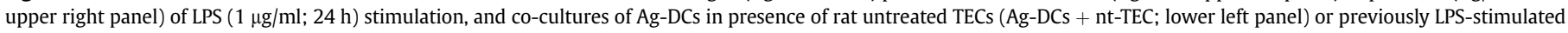

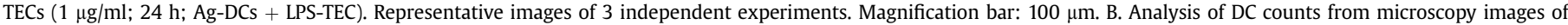

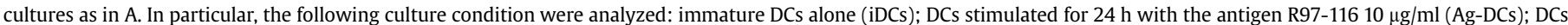

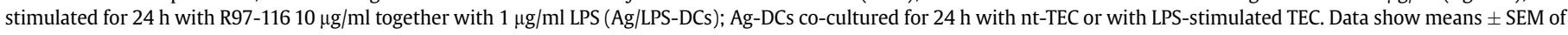

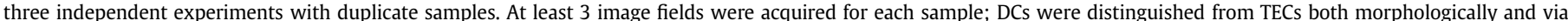

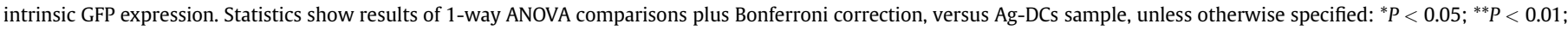

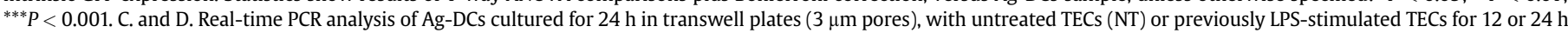

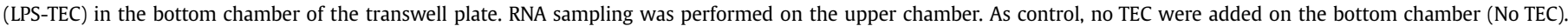

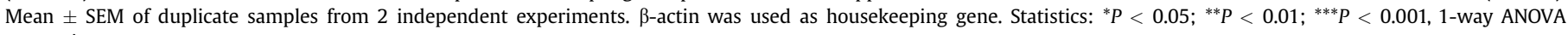
comparisons.

an increased meandering index. These data suggest that in the complex thymic milieu fine interactions and cell-to-cell contacts may occur, dampening Teff chance to form stable synapses with DCs.

To evaluate whether an activated innate immunity microenvironment may influence Teff motility and the chances of immunological synapse formation, we added LPS-stimulated TECs to Teffs/ Ag-DCs co-culture (Fig. 8D; Supplementary Video 4). TLR4activated TECs led to a sharp decrease in Teff $V_{\max }$ in the first 20min co-culture, related to a steep increase in the percentage of Teff stationary cells and equal decrease of Teff motile cells, showing low meandering index (Table 3). Thus, TEC LPS-stimulation facilitated cell-to-cell contacts between Teffs and DCs, with the latter showing a more pronounced activated morphology (Fig. 8D; Supplementary Video 4). When Tregs were added to LPS-stimulated TECs/Teffs/Ag-DCs co-culture, their modulatory effect was lost: indeed, most Teffs quickly acquired a stationary phenotype (60\% at 30-min; Table 3) with abruptly dropped Teff $V_{\max }$ (Fig. 8E; Supplementary Video 5). Nevertheless, Teff motile cells remained more than $10 \%$ even after 90 -min co-culture (Supplementary Fig. 5), with a relatively higher displacement, sign of random movement.

\subsection{The inflamed microenvironment might favor autoreactive Teff activation}

To finely assess activation of AChR-specific Teff, we analyzed $\left[\mathrm{iCa}^{2+}\right]$ level and showed that Teff $\left[\mathrm{iCa}^{2+}\right]$ quickly increased in all co-culture conditions, consistent with the formation of TCR/AgMHC contacts. The presence of Tregs significantly diminished Teff $\left[\mathrm{iCa}^{2+}\right]$ (Fig. 9A and B). LPS-stimulated TECs (LPS-TECs), but not untreated TECs (nt-TECs), increased Teff $\left[\mathrm{iCa}^{2+}\right]$ both in the absence and in the presence of Tregs (Fig. 9A and B). However, after the initial Teff $\left[\mathrm{iCa}^{2+}\right]$ spike, Tregs were able to reduce the level of $\left[\mathrm{iCa}^{2+}\right]$ plateau, probably due to balancing contacts between Tregs and Teffs or Tregs and Ag-DCs (Fig. 9B).

Finally, we evaluated the inflammatory cytokine profile of the different co-culture set-ups, compared with that of Teffs and Tregs single cultures (Fig. 9C). IL17A and IFN $\gamma$ mRNA levels confirmed 
A
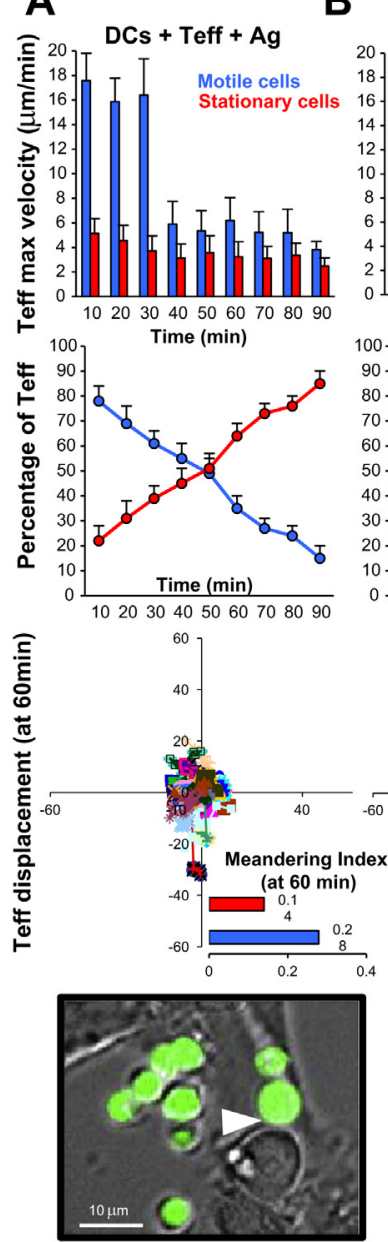

B

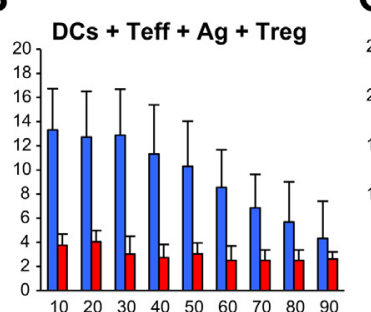

$10 \begin{array}{lllllllll}10 & 30 & 40 & 50 & 60 & 70 & 80 & 90\end{array}$
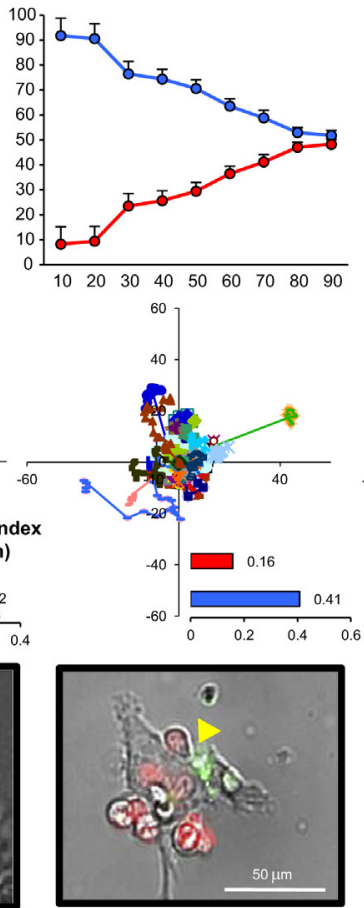

C
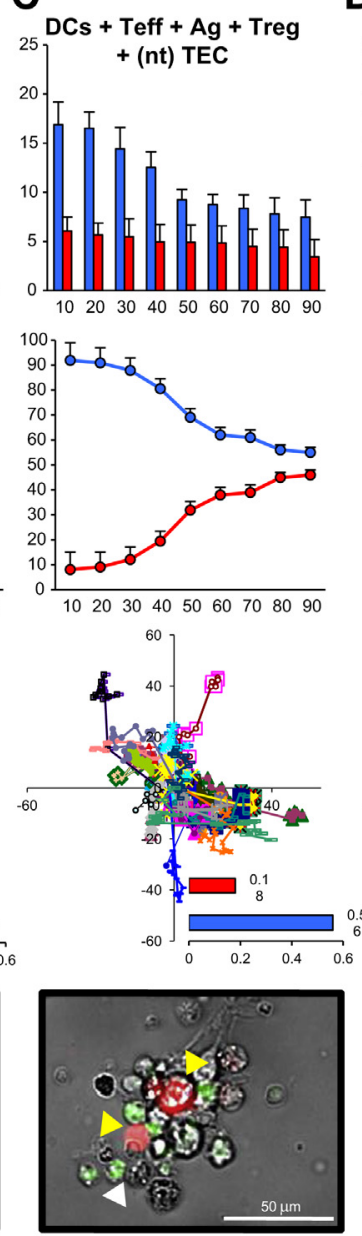

D
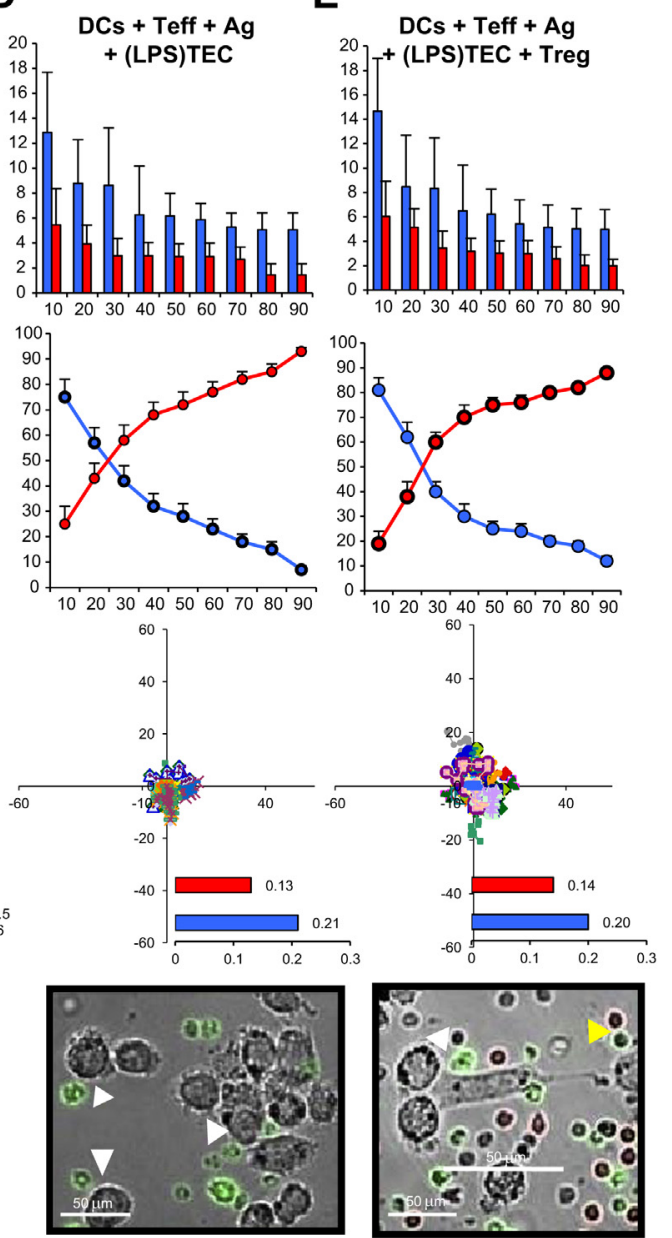

$\mathbf{E}$
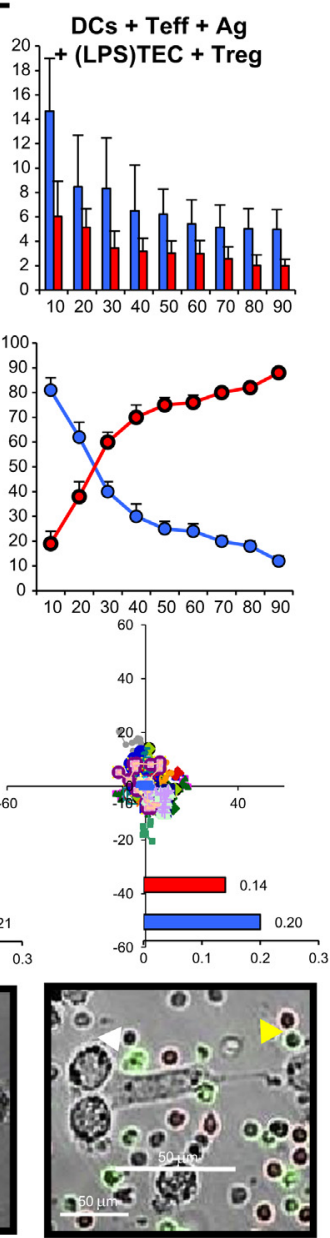

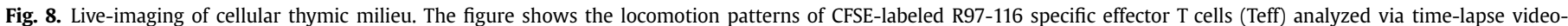

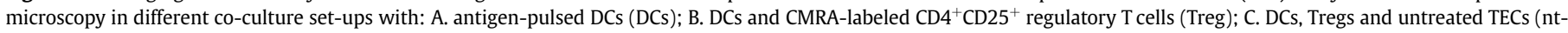

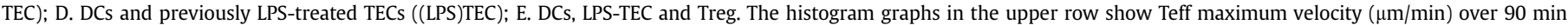

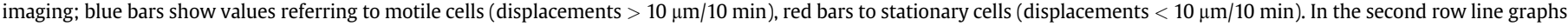

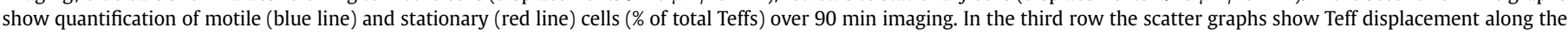

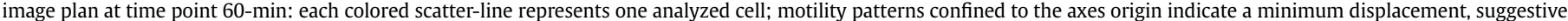

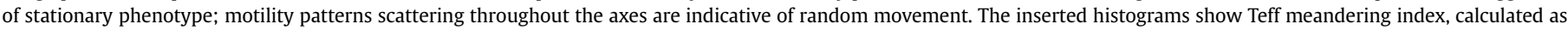

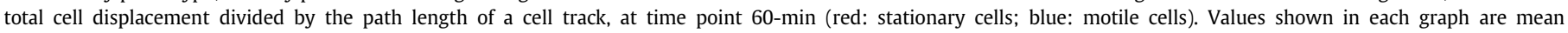

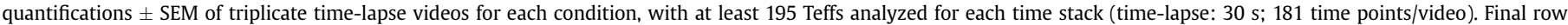

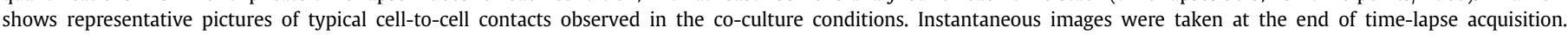

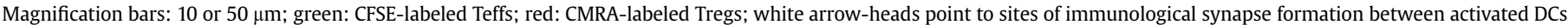
and Teffs; yellow arrow-heads indicate contacts with Tregs.

the Th1/Th17 phenotype of R97-116 specific Teffs, whereas the levels of FoxP3 and TGF- $\beta$ confirmed the regulatory phenotype of naïve Tregs; IL-2R $\alpha$ mRNA levels were quite high not only in Tregs but also in Teffs, as previously shown [33]. A marked CCR4 mRNA expression was observed following $12 \mathrm{~h}$ Teffs/Ag-DCs co-culture, accompanied by high levels of IL17A, IFN- $\gamma$, and TNF- $\alpha$ mRNAs. These activatory markers, together with CCR4, were instead downregulated in Treg/Teffs/Ag-DCs co-culture. Of note, the presence of LPS-stimulated TECs determined a sharp increase in CCL17 and CCL22 mRNA expression, accompanied by reduced CCR4, suggestive of post-activatory receptor down-regulation. Similarly, IL-2R $\alpha$, IFN- $\gamma$, and IL17A mRNAs were down-regulated, while TNF- $\alpha$ was up-regulated. Tregs in the co-culture only partially attenuated the effect induced by LPS-TECs: the levels of CCL17, IL-17, IFN- $\gamma$, and TNF- $\alpha$, but not CCL22, were less abundant (Fig. 9C). Overall, these data suggest that the TLR4-activated TEC microenvironment may result in an altered balance of cytokines and chemokines network.

\section{Discussion}

The thymus is the main site of autoimmunity development and maintenance in AChR-MG. Accumulating data show that chronic inflammation and innate immune activation are key features of hyperplastic MG thymus [34], pushing the need to better investigate and identify the molecular mechanisms linking the long-term inflammatory state with the autoimmune process. Abnormal recruitment of immune cells and altered Treg-mediated immunoregulation are possible mechanisms responsible for sustaining inflammation and immune response to AChR in MG thymus.

Chemokines and their receptors are key elements in the development and maintenance of innate and adaptive immune responses, orchestrating homing and interactions of DCs, T and B cells into inflammatory sites and secondary lymphoid organs [10]. CXCL13, CCL21, CCL19 and CXCL10 have been demonstrated to be up-regulated in MG thymus, thus suggesting a role in MG thymic 
Table 3

Motility characteristics of AChR-specific effector T cells in TLR4-stimulated thymic milieu.

\begin{tabular}{|c|c|c|c|}
\hline 30 min co-culture & \% Motile cells & Max. velocity of motile cells & Meandering index of motile cells \\
\hline $\mathrm{DC}+\mathrm{Teff}+\mathrm{Ag}$ & $61 \pm 5$ & $16.40 \pm 2.95$ & $0.43 \pm 0.15$ \\
\hline $\mathrm{DC}+$ Teff $+\mathrm{Ag}+$ Treg & $76 \pm 9^{*}$ & $12.88 \pm 3.80$ & $0.39 \pm 0.21$ \\
\hline $\mathrm{DC}+$ Teff + Ag + Treg $+(n t)$ TEC & $88 \pm 5^{* * *}$ & $14.42 \pm 2.17$ & $0.69 \pm 0.15^{*}$ \\
\hline $\mathrm{DC}+\mathrm{Teff}+\mathrm{Ag}+\mathrm{LPS}) \mathrm{TEC}$ & $42 \pm 6^{* *}$ & $8.63 \pm 4.60^{* *}$ & $0.63 \pm 0.17^{*}$ \\
\hline $\begin{array}{l}\mathrm{DC}+\mathrm{Teff}+\mathrm{Ag}+\text { Treg }+(\text { LPS }) \mathrm{TEC} \\
60 \text { min co-culture }\end{array}$ & $40 \pm 4^{* *} \S \S \S$ & $8.32 \pm 4.16^{* *} \S$ & $0.60 \pm 0.17$ \\
\hline $\mathrm{DC}+$ Teff $+\mathrm{Ag}$ & $35 \pm 5$ & $5.20 \pm 1.58$ & $0.28 \pm 0.08$ \\
\hline $\mathrm{DC}+$ Teff $+\mathrm{Ag}+$ Treg & $64 \pm 6^{* * *}$ & $8.55 \pm 3.11$ & $0.41 \pm 0.06$ \\
\hline $\mathrm{DC}+$ Teff + Ag + Treg $+(n t)$ TEC & $62 \pm 3^{* * *}$ & $8.74 \pm 1.04$ & $0.56 \pm 0.14^{*}$ \\
\hline $\mathrm{DC}+$ Teff $+\mathrm{Ag}+($ LPS $)$ TEC & $23 \pm 4^{*}$ & $5.87 \pm 1.29$ & $0.21 \pm 0.08$ \\
\hline $\begin{array}{l}\mathrm{DC}+\mathrm{Teff}+\mathrm{Ag}+\text { Treg }+(\mathrm{LPS}) \mathrm{TEC} \\
90 \text { min co-culture }\end{array}$ & $24 \pm 3^{*} \S \S$ & $5.41 \pm 1.95$ & $0.20 \pm 0.08 \S \S \S$ \\
\hline $\mathrm{DC}+$ Teff $+\mathrm{Ag}$ & $15 \pm 5$ & $3.83 \pm 0.66$ & $0.21 \pm 0.09$ \\
\hline $\mathrm{DC}+$ Teff $+\mathrm{Ag}+$ Treg & $52 \pm 4^{* * *}$ & $4.33 \pm 3.09$ & $0.29 \pm 0.03$ \\
\hline $\mathrm{DC}+$ Teff + Ag + Treg $+(n t)$ TEC & $55 \pm 2^{* * *}$ & $7.46 \pm 1.76$ & $0.54 \pm 0.12^{* *}$ \\
\hline $\mathrm{DC}+\mathrm{Teff}+\mathrm{Ag}+($ LPS $) \mathrm{TEC}$ & $7 \pm 2$ & $5.06 \pm 1.35$ & $0.15 \pm 0.06$ \\
\hline DC + Teff + Ag + Treg + (LPS)TEC & $12 \pm 2 \S \S \S$ & $4.98 \pm 1.62 \S \S$ & $0.15 \pm 0.06 \S \S \S$ \\
\hline
\end{tabular}

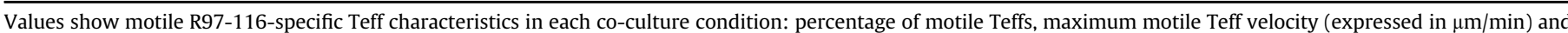

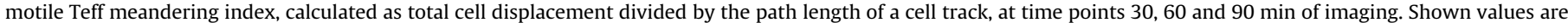

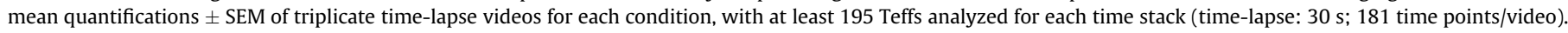

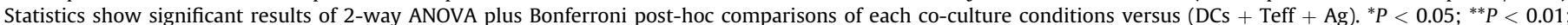

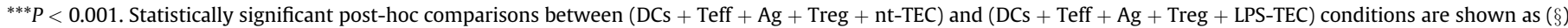
$P<0.05$ and $(\S \S) P<0.05$.

pathology and etiology, via peripheral T- and B-cell recruitment and lymphoid formation [3,6]. Migration of peripheral immune cells in MG thymus and hyperplastic changes could be also favored by the abnormal development of lymphatic vessels and HEVs [35]. Recently, HEVs were found to express SDF-1, a chemokine supposed to drive the migration of CXCR4 ${ }^{+}$APCs and B cells to the thymus and to participate in hyperplastic changes [36].

Other chemokines may be involved in thymic MG pathogenesis, such as CCL17 and CCL22. They share the CCR4 receptor [14,15], expressed on functionally distinct subsets of T cells, such as Th2, Th17, Treg cells, and DCs [11,17,37]; the latter are also able to produce CCL17 and CCL22 [12,13]. This chemokine/chemokine receptor axis has been implicated in autoimmune models [38] and in human autoimmune conditions, such as multiple sclerosis [39] and rheumatoid arthritis [40].

Herein, we hypothesized a role of CCR4 and its ligands in driving abnormal recruitment of peripheral immune cells to MG thymus. The significant increase of CCL17, CCL22 and CCR4 in hyperplastic MG thymuses (Figs. 1 and 4) suggests that these chemokines might play a crucial role in thymic MG etiology. Importantly, in MG thymuses CCL17 and CCL22 were mainly expressed by medullary HCs and surrounding TECs and DCs, as confirmed by laser-capture microdissection (Figs. 1 and 2). HCs were previously described as sites of CCL22 production [41]: CCL22 is expressed by a distinct subset of medullary TECs localized to the outer part of HCs and is one of the chemokines responsible for organizing thymocyte populations into discrete microenvironments, an important process in the negative and positive selections of T-cells [41]. HCs also produce IL-17, SDF-1 and thymic stromal lymphopoietin, the latter able to stimulate thymic DCs in producing high levels of CCL17 and CCL22 [42].

Our studies co-localized CCL17 and CCL22 with TLR4 in TECs and DCs associated with HCs present in MG thymuses (Fig. 2), and allowed us to hypothesize that TLR4-mediated signaling might contribute to abnormal expression and production of the two chemokines in MG thymus. We confirmed this via in vitro studies on TECs isolated from MG and EAMG thymuses, showing a significant increase of CCL17 and CCL22 expression after TLR4 stimulation with LPS (Figs. 4 and 6).

In MG thymuses, CCL17 was also expressed in HEVs (Fig. 1D). Our observation that corticosteroid treatment tended to normalize transcriptional levels of CCL17, but not of CCL22 (Fig. 4), could be due to the normalization of the number of HEVs in corticosteroidtreated MG thymus [36].

CCR4 is known to drive the migration of DCs and Tregs into antigen presentation sites in inflamed areas $[16,17]$. The observed increased proportion of DC-LAMP ${ }^{+}$mature DCs, and more specifically of CCR4 ${ }^{+}$DC-LAMP $^{+}$DCs, in MG compared to normal thymic sections (Fig. 3), suggests an enhanced $\mathrm{CCR}^{+}$DCs migration to inflamed MG thymus. CCR4 ${ }^{+}$DC-LAMP $^{+}$DCs mainly localized around HCs, indicating an active role for HC-produced CCL17 and CCL22 in homing CCR4 ${ }^{+}$DCs around medullary HCs, where these cells could be induced to maturate and therefore express DC-LAMP [42]. This event might start a feedback loop of DC infiltration, via a further CCL17/CCL22 production by DCs.

Published data demonstrated the involvement of CCR4 and its ligands on APC functions: binding of antigen-primed T cells to activated DCs was found to be dependent on CCR4 and the duration of their cognate interaction correlated with increased CCL17 production by DCs $[43,44]$. As CCR4 ${ }^{+}$mature DCs were increased in MG thymuses, we may speculate that these cells could cross-present the autoantigen to AChR-specific T cells, thus favoring maintenance of the inflammatory autoimmune process.

As far as Tregs are concerned, we neither found differences in the number of $\mathrm{FoxP}^{+}$Tregs nor in the percentage of $\mathrm{CCR} 4^{+}{ }^{+} \mathrm{FoxP} 3^{+}$ cells between MG thymuses and controls, in line with previous data [45]. However, Treg suppressive function is severely defective in MG thymus [9], and studies in the EAMG model showed that disequilibrium of Th1/Th2/Th17 effector cells and Tregs, and abnormal expression of cytokines affecting Th1/Th2/Th17 balance, may be implicated in MG pathogenesis [46]. The mechanisms underlying Treg dysfunction in MG thymus are still matter of debate; in particular, it is unclear whether Treg impairment is a primary event in MG pathogenesis, or a consequence of the inflammatory thymic microenvironment.

Since innate immune system activation significantly affects cytokine networks, we hypothesized that TLR4 signaling in MG thymus could alter the expression of cytokines involved in Treg function and Teff/Treg balance. Th17-related cytokines are known to play a key role in chronic inflammation and autoimmunity [47] and are mainly secreted by T-cells. However, considerable data suggest that initiation of IL-17-dependent inflammatory responses 


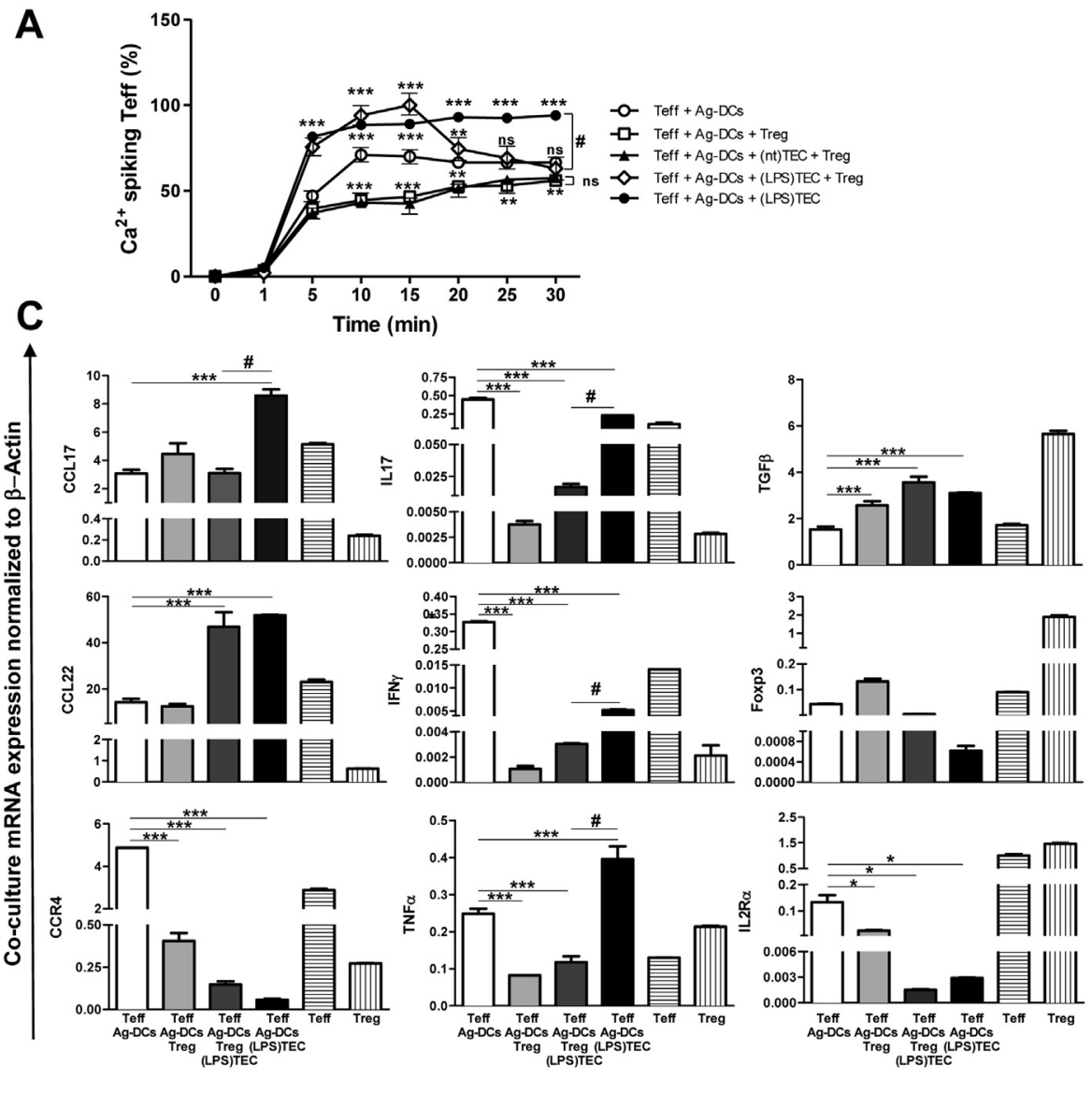

B
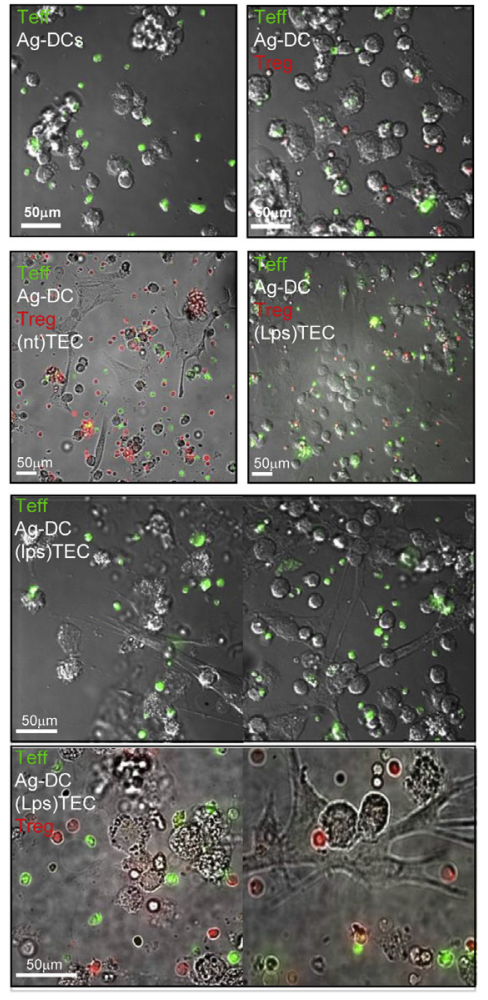

Culture condition

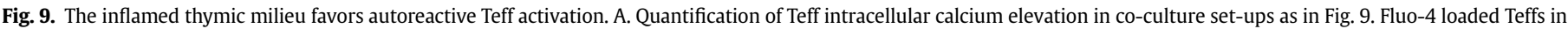

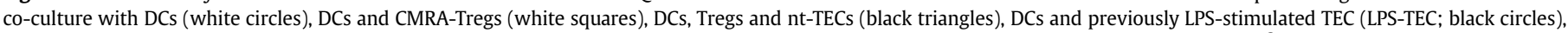

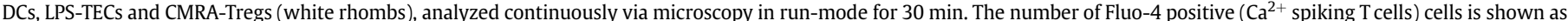

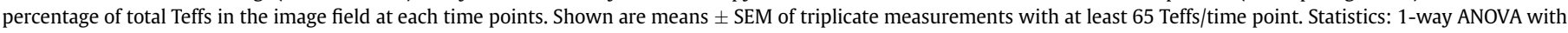

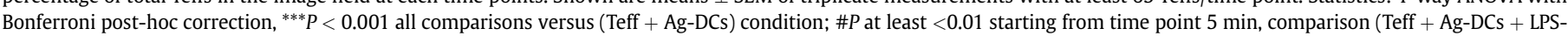

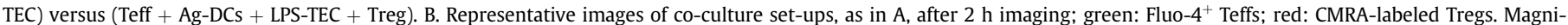

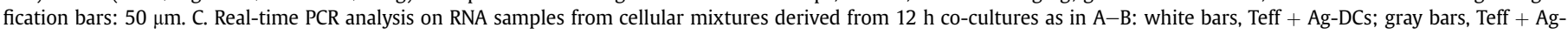

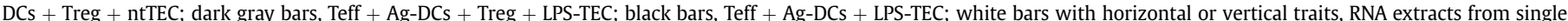

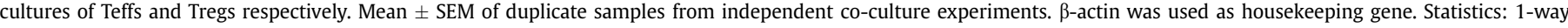

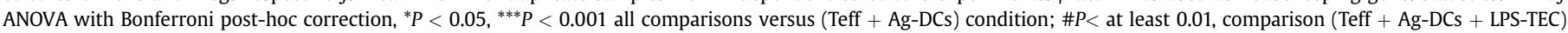
versus (Teff + Ag-DCs + LPS-TEC + Treg).

to stress, injury or pathogens involves innate immune cell populations, including invariant natural killer T-cells, lymphoid-tissue inducer-like cells, NK cells, myeloid cells and epithelial cells, in tissues specialized in immune surveillance [48]. TECs from MG patients are known to produce high levels of IL- 6 and IL-1 $\beta$ [3], two cytokines of the Th17-related family that may affect Treg function by driving FoxP3 ${ }^{+}$cells to secrete IL-17 [49]. We confirmed production of IL- 6 and IL1 $\beta$ by MG TECs both in basal condition and after TLR4 stimulation (Table 2). Moreover, we found a significant increase of IL-4, IFN- $\gamma$, and IL-17F secretion by TECs stimulated with LPS for $48 \mathrm{~h}$. As IL-17F and IL-17A share the IL-17RA/RC receptor and exert common biological effects [48], we suggest that IL-17F produced by MG TECs could affect immunoregulation in MG thymus. Indeed, we found that IL-17F, IL-17A and IL-6 mRNA levels were significantly increased in hyperplastic MG thymuses, accompanied by a decreased expression of TGF- $\beta$ (Fig. 5), further supporting that Th17-related cytokine balance may contribute to inflammation and immunodysregulation occurring in MG thymus.

Our results seem to indicate the thymic microenvironment surrounding HCs as a privileged site for AChR autosensization.
Indeed, all the important players in the anti-AChR autoimmune response are present into restricted medullary areas encircling MG HCs: 1) TECs and myoid cells, known to express the autoantigen [50,51]; 2) mature DC-LAMP ${ }^{+}$myeloid DCs [3], that may crosspresent the autoantigen; 3) B-cells, able to produce anti-AChR antibodies [52], following activation by 4 ) autoreactive Teffs [53]; 5) $\mathrm{FoxP}^{+}$Tregs, which are likely inefficient in dampening the autoimmune response [9]; and 6) HEVs, representing the entry site for migrating CCR4 ${ }^{+}$DCs [54]. Close interactions between Teffs and APCs at the medullary HC areas might play a critical role in initiation and maintenance of AChR-specific immune responses.

The best way to observe close interactions among immune cells is certainly in vivo imaging of the immune tissue, as recently reviewed [55]. T-cell adaptive immune responses [56,57] and humoral immunity induction [58] as well as the immune reactions in response to pathogens [59] and the effector functions of autoreactive encephalitogenic T cells [60] have been extensively investigated by in vivo imaging. Unfortunately, due to its anatomical position and adulthood-induced size reduction, the thymus is an organ not feasibly accessible by in vivo microscopy and, to our 


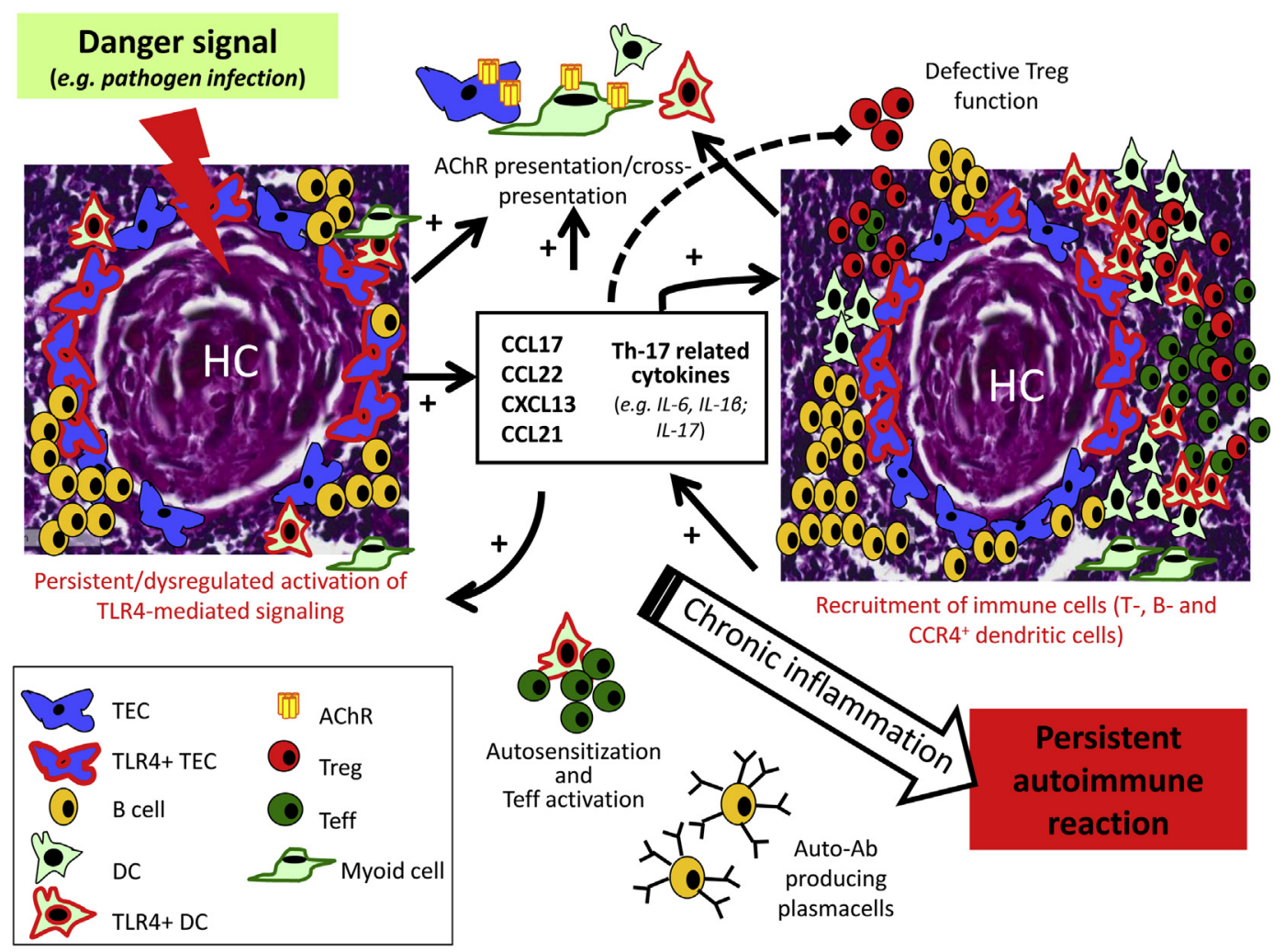

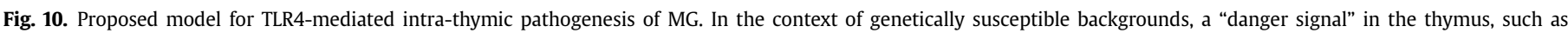

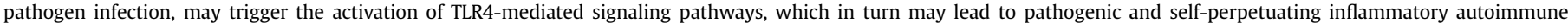

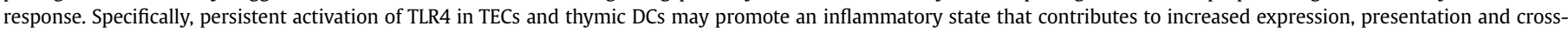

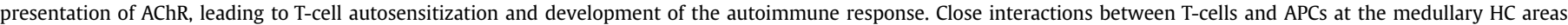

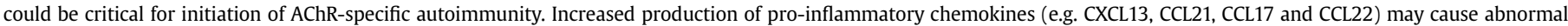

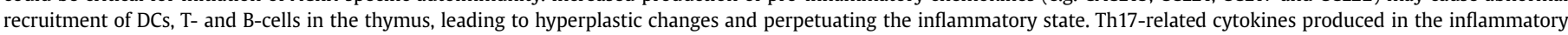

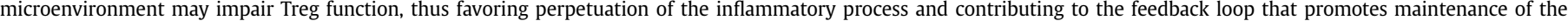
autoimmune response in MG thymus.

knowledge, no reports on direct in vivo imaging of the thymus have been published. Therefore, we developed an in vitro model to mimic the microenvironment of thymic $\mathrm{HCs}$, consisting of naïve or EAMG rat-derived TEC and DC primary cultures, and as effector cells AChR $\alpha$-subunit specific Th1/Th17 lymphocytes [21]. In this model Teffs moved randomly (motile cells) until encountering the cognate antigen presented by Ag-DCs, at which point they slowed down and remained fixed to anchor sites (stationary cells), forming a relatively stable immunological synapse (Fig. 8A; Supplementary Video 1). Our in vitro co-culture set-up was able to reproduce the in vivo cell-to-cell contacts between Teffs and DCs, with timing and cell movement velocities being comparable to published data on models in live animals [26,30]. When naïve rat-derived $\mathrm{CD}_{127}{ }^{-} \mathrm{CD} 4^{+} \mathrm{CD} 25^{+}$Tregs were added to our co-culture system, a reduced rate of stable contact formation between Teffs and DCs was observed, quantified by a minor percentage of stationary cells and an increased cellular displacement (Fig. 8B; Supplementary Video 2 ). As suggested elsewhere [31,32], this effect was likely due to physical competition between Tregs and Teffs in forming contacts with DCs, and even more amplified when a fourth cellular partner, i.e. untreated TECs, was added (Fig. 8C; Supplementary Video 3). Indeed, the co-presence of multiple cellular counterparts dampened the chances of immunological synapse formation between autoreactive Teff cells and activated DCs.

As recently reported [61], minor Teff/DC stable contacts correlated with reduced Teff calcium signals. The quick and steep elevation in Teff intracellular calcium, observed in control Teff/DC co-culture, was significantly diminished when Tregs or Tregs/ntTECs were added in our cellular model (Fig. 9A and B). These data suggest an effective role of naïve unspecific Tregs in attenuating specific Teff activation in a thymic microenvironment culture setup. However, innate immunity activation via LPS-TLR4 engagement on TECs, significantly impaired Treg ability, as visualized by diminished Teff motility/displacement (Fig. 8E) and increased Teff calcium signaling (Fig. 9A and B). This is in line with published data showing LPS effect in dampening Treg functions [62], whilst inducing Th1- or Th2-specific enhanced responses [63]. Importantly, the presence of LPS-stimulated TECs induced DC activation and proliferation (Fig. 7), reminiscent of direct LPS/TLR4 ligation on DCs [64], which can account for DC increased antigen presenting capacity, leading to more stable contacts with Teffs (Supplementary Videos 4 and 5) and minor Treg suppressive capacity, in agreement with previous data [65]. Thus, in our in vitro inflamed model, the AChR-specific immune reaction is aided and sped-up by increased numbers of pro-activatory CCR4 ${ }^{+}$DCs and dampened Treg suppressive capacity. This leads to a general pro-inflammatory milieu, characterized by high IL-17, IFN- $\gamma$, TNF- $\alpha$ expression levels, accompanied by leukocyte trafficking inducing chemokines such as CCL17 and CCL22 (Fig. 9C), functionally resembling MG thymic inflamed microenvironment. In such activated microenvironment, we may speculate an easy B cell differentiation into anti-AChR antibody producing plasma cells, aided by activated Teffs and follicular $\mathrm{OX}_{40} 0^{+} \mathrm{CD}_{40 \mathrm{~L}}{ }^{+}$Th cells [66]. In this respect, new evidence has recently come out on the role of follicular Th cells both in MG 
patients [67] and in the EAMG model [68], suggesting these cells as additional players in immune cell network, that triggers and perpetuates intrathymic autoimmunity.

\section{Conclusions}

Increasing data indicate that chronic inflammation and innate immune activation via TLRs characterize MG thymus. The overall findings here presented suggest a role for TLR4 signaling in driving immune cell recruitment in MG thymus and in generating an inflammatory response, that in turn may disrupt immunoregulatory mechanisms, favoring autosensitization and autoimmunity. Our results prompted us to propose a model of intra-thymic MG pathogenesis, in which persistent TLR4 signaling, abnormal recruitment of DCs, and altered Th17-related cytokine network impairing Treg function, are key events for the initiation and perpetuation of the inflammatory autoimmune process within MG thymus (Fig. 10).

\section{Disclosures}

None of the authors have any financial conflict of interest.

\section{Acknowledgments}

We thank Drs. Antonio Lanzavecchia and Federica Sallusto (Institute for Research in Biomedicine, Bellinzona, $\mathrm{CH}$ ) for kind gift of cyanobacterial LPS antagonist (CyP); Dr. Renato Longhi (CNR, Istituto di Chimica del Riconoscimento Molecolare, Milan, Italy) for R97-116 peptide synthesis; Dr. Naoto Kawakami (MPI-Neurobiology, Muenchen, Germany) for providing the colony founders of EGFP-transgenic rats. This work was supported by 7th Framework Programme of the European Union FIGHT-MG (Grant No. 242210) and by Italian Ministry of Health, year 2012 (annual research funding).

\section{Appendix A. Supplementary data}

Supplementary data related to this article can be found online at http://dx.doi.org/10.1016/j.jaut.2013.12.013.

\section{References}

[1] Cavalcante P, Bernasconi P, Mantegazza R. Autoimmune mechanisms in myasthenia gravis. Curr Opin Neurol 2012;25:621-9.

[2] Marx A, Pfister F, Schalke B, Saruhan-Direskeneli G, Melms A, Strobel P. The different roles of the thymus in the pathogenesis of the various myasthenia gravis subtypes. Autoimmun Rev 2013;12:875-84.

[3] Cavalcante P, Le Panse R, Berrih-Aknin S, Maggi L, Antozzi C, Baggi F, et al. The thymus in myasthenia gravis: site of "innate autoimmunity"? Muscle Nerve 2011;44:467-84.

[4] Bernasconi P, Barberis M, Baggi F, Passerini L, Cannone M, Arnoldi E, et al Increased toll-like receptor 4 expression in thymus of myasthenic patients with thymitis and thymic involution. Am J Pathol 2005;167:129-39.

[5] Cufi P, Dragin N, Weiss JM, Martinez-Martinez P, De Baets MH, Roussin R, et al. Implication of double-stranded RNA signaling in the etiology of autoimmune myasthenia gravis. Ann Neurol 2013;73:281-93.

[6] Cavalcante P, Cufi P, Mantegazza R, Berrih-Aknin S, Bernasconi P, Le Panse R. Etiology of myasthenia gravis: innate immunity signature in pathological thymus. Autoimmun Rev 2013;12:863-74.

[7] Kurt-Jones EA, Popova L, Kwinn L, Haynes LM, Jones LP, Tripp RA, et al. Pattern recognition receptors TLR4 and CD14 mediate response to respiratory syncytial virus. Nat Immunol 2000;1:398-401.

[8] Cavalcante P, Barberis M, Cannone M, Baggi F, Antozzi C, Maggi L, et al. Detection of poliovirus-infected macrophages in thymus of patients with myasthenia gravis. Neurology 2010;74:1118-26.

[9] Gradolatto A, Nazzal D, Foti M, Bismuth J, Truffault F, Le Panse R, et al. Defects of immunoregulatory mechanisms in myasthenia gravis: role of IL-17. Ann N Y Acad Sci 2012:1274:40-7.

[10] Sallusto F, Lanzavecchia A, Mackay CR. Chemokines and chemokine receptors in T-cell priming and Th1/Th2-mediated responses. Immunol Today 1998;19: 568-74.
[11] Imai T, Nagira M, Takagi S, Kakizaki M, Nishimura M, Wang J, et al. Selective recruitment of CCR4-bearing Th2 cells toward antigen-presenting cells by the CC chemokines thymus and activation-regulated chemokine and macrophage-derived chemokine. Int Immunol 1999;11:81-8.

[12] Vulcano M, Albanesi C, Stoppacciaro A, Bagnati R, D’Amico G, Struyf S, et al. Dendritic cells as a major source of macrophage-derived chemokine/CCL22 in vitro and in vivo. Eur J Immunol 2001;31:812-22.

[13] Penna G, Vulcano M, Sozzani S, Adorini L. Differential migration behavior and chemokine production by myeloid and plasmacytoid dendritic cells. Hum Immunol 2002;63:1164-71.

[14] Imai T, Baba M, Nishimura M, Kakizaki M, Takagi S, Yoshie O. The T celldirected CC chemokine TARC is a highly specific biological ligand for CC chemokine receptor 4. J Biol Chem 1997;272:15036-42.

[15] Imai T, Chantry D, Raport CJ, Wood CL, Nishimura M, Godiska R, et al. Macrophage-derived chemokine is a functional ligand for the CC chemokine receptor 4. J Biol Chem 1998:273:1764-8.

[16] Yuan Q, Bromley SK, Means TK, Jones KJ, Hayashi F, Bhan AK, et al. CCR4dependent regulatory $\mathrm{T}$ cell function in inflammatory bowel disease. J Exp Med 2007;204:1327-34.

[17] Stutte S, Quast T, Gerbitzki N, Savinko T, Novak N, Reifenberger J, et al. Requirement of CCL17 for CCR7- and CXCR4-dependent migration of cutaneous dendritic cells. Proc Natl Acad Sci U S A 2010;107:8736-41.

[18] Fu W, Chen W. Roles of chemokines in thymopoiesis: redundancy and regulation. Cell Mol Immunol 2004;1:266-73.

[19] Waite JC, Skokos D. Th17 response and inflammatory autoimmune diseases. Int J Inflam 2012;2012:819467.

[20] Baggi F, Antozzi C, Toscani C, Cordiglieri C. Acetylcholine receptor-induced experimental myasthenia gravis: what have we learned from animal models after three decades? Arch Immunol Ther Exp (Warsz) 2012;60:19-30.

[21] Baggi F, Annoni A, Ubiali F, Milani M, Longhi R, Scaioli W, et al. Breakdown of tolerance to a self-peptide of acetylcholine receptor alpha-subunit induces experimental myasthenia gravis in rats. J Immunol 2004;172:2697-703.

[22] Inoue H, Ohsawa I, Murakami T, Kimura A, Hakamata Y, Sato Y, et al. Development of new inbred transgenic strains of rats with LacZ or GFP. Biochem Biophys Res Commun 2005;329:288-95.

[23] Macagno A, Molteni M, Rinaldi A, Bertoni F, Lanzavecchia A, Rossetti C, et al. A cyanobacterial LPS antagonist prevents endotoxin shock and blocks sustained TLR4 stimulation required for cytokine expression. J Exp Med 2006;203:1481-92.

[24] Marolda R, Ruocco C, Cordiglieri C, Toscani C, Antozzi C, Mantegazza R, et al. Differential targeting of immune-cells by Pixantrone in experimental myasthenia gravis. J Neuroimmunol 2013;258:41-50.

[25] Flugel A, Willem M, Berkowicz T, Wekerle H. Gene transfer into CD4+ T lymphocytes: green fluorescent protein-engineered, encephalitogenic T cells illuminate brain autoimmune responses. Nat Med 1999;5:843-7.

[26] Kawakami N, Nagerl UV, Odoardi F, Bonhoeffer T, Wekerle H, Flugel A. Live imaging of effector cell trafficking and autoantigen recognition within the unfolding autoimmune encephalomyelitis lesion. J Exp Med 2005;201:1805-14.

[27] Cordiglieri C, Odoardi F, Zhang B, Nebel M, Kawakami N, Klinkert WE, et al. Nicotinic acid adenine dinucleotide phosphate-mediated calcium signalling in effector T cells regulates autoimmunity of the central nervous system. Brain 2010;133:1930-43.

[28] Parker LC, Whyte MK, Vogel SN, Dower SK, Sabroe I. Toll-like receptor (TLR)2 and TLR4 agonists regulate CCR expression in human monocytic cells. J Immunol 2004;172:4977-86.

[29] Miller MJ, Hejazi AS, Wei SH, Cahalan MD, Parker I. T cell repertoire scanning is promoted by dynamic dendritic cell behavior and random $\mathrm{T}$ cell motility in the lymph node. Proc Natl Acad Sci U S A 2004;101:998-1003.

[30] Miller MJ, Safrina O, Parker I, Cahalan MD. Imaging the single cell dynamics of CD4+ T cell activation by dendritic cells in lymph nodes. J Exp Med 2004;200: $847-56$.

[31] Tadokoro CE, Shakhar G, Shen S, Ding Y, Lino AC, Maraver A, et al. Regulatory T cells inhibit stable contacts between CD4 + T cells and dendritic cells in vivo. J Exp Med 2006;203:505-11.

[32] Tomiyama T, Ueda Y, Katakai T, Kondo N, Okazaki K, Kinashi T, Antigenspecific suppression and immunological synapse formation by regulatory $\mathrm{T}$ cells require the mst1 kinase. PLoS One 2013;8:e73874.

[33] Flugel A, Berkowicz T, Ritter T, Labeur M, Jenne DE, Li Z, et al. Migratory activity and functional changes of green fluorescent effector cells before and during experimental autoimmune encephalomyelitis. Immunity 2001;14: 547-60.

[34] Le Panse R, Berrih-Aknin S. Autoimmune myasthenia gravis: autoantibody mechanisms and new developments on immune regulation. Curr Opin Neurol 2013;26:569-76.

[35] Le Panse R, Bismuth J, Cizeron-Clairac G, Weiss JM, Cufi P, Dartevelle P, et al. Thymic remodeling associated with hyperplasia in myasthenia gravis. Autoimmunity 2010;43:401-12.

[36] Weiss JM, Cufi P, Bismuth J, Eymard B, Fadel E, Berrih-Aknin S, et al. SDF-1/ CXCL12 recruits $B$ cells and antigen-presenting cells to the thymus of autoimmune myasthenia gravis patients. Immunobiology 2013;218:373-81.

[37] Lim HW, Lee J, Hillsamer P, Kim CH. Human Th17 cells share major trafficking receptors with both polarized effector T cells and FOXP3+ regulatory T cells. Immunol 2008;180:122-9.

[38] Poppensieker K, Otte DM, Schurmann B, Limmer A, Dresing P, Drews E, et al. CC chemokine receptor 4 is required for experimental autoimmune 
encephalomyelitis by regulating GM-CSF and IL-23 production in dendritic cells. Proc Natl Acad Sci U S A 2012;109:3897-902.

[39] Galimberti D, Fenoglio C, Comi C, Scalabrini D, De Riz M, Leone M, et al. MDC/ CCL22 intrathecal levels in patients with multiple sclerosis. Mult Scler 2008; 14:547-9.

[40] Flytlie HA, Hvid M, Lindgreen E, Kofod-Olsen E, Petersen EL, Jorgensen A, et al. Expression of MDC/CCL22 and its receptor CCR4 in rheumatoid arthritis, psoriatic arthritis and osteoarthritis. Cytokine 2010;49:24-9.

[41] Annunziato F, Romagnani P, Cosmi L, Lazzeri E, Romagnani S. Chemokines and lymphopoiesis in human thymus. Trends Immunol 2001;22:277-81.

[42] Watanabe N, Wang YH, Lee HK, Ito T, Wang YH, Cao W, et al. Hassall's corpuscles instruct dendritic cells to induce CD4+CD25+ regulatory $\mathrm{T}$ cells in human thymus. Nature 2005;436:1181-5.

[43] Wu M, Fang H, Hwang ST. Cutting edge: CCR4 mediates antigen-primed T cell binding to activated dendritic cells. J Immunol 2001;167:4791-5.

[44] Henry CJ, Ornelles DA, Mitchell LM, Brzoza-Lewis KL, Hiltbold EM. IL-12 produced by dendritic cells augments CD8 + T cell activation through the production of the chemokines CCL1 and CCL17. J Immunol 2008;181:8576-84.

[45] Matsui N, Nakane S, Saito F, Ohigashi I, Nakagawa Y, Kurobe H, et al. Undiminished regulatory $\mathrm{T}$ cells in the thymus of patients with myasthenia gravis. Neurology 2010;74:816-20.

[46] Gertel-Lapter S, Mizrachi K, Berrih-Aknin S, Fuchs S, Souroujon MC. Impairment of regulatory $\mathrm{T}$ cells in myasthenia gravis: studies in an experimental model. Autoimmun Rev 2013;12:894-903.

[47] Hemdan NY, Birkenmeier G, Wichmann G, Abu El-Saad AM, Krieger T, Conrad $\mathrm{K}$, et al. Interleukin-17-producing $\mathrm{T}$ helper cells in autoimmunity. Autoimmun Rev 2010;9:785-92.

[48] Cua DJ, Tato CM. Innate IL-17-producing cells: the sentinels of the immune system. Nat Rev Immunol 2010;10:479-89.

[49] Voo KS, Wang YH, Santori FR, Boggiano C, Wang YH, Arima K, et al. Identification of IL-17-producing FOXP3 + regulatory T cells in humans. Proc Natl Acad Sci U S A 2009;106:4793-8.

[50] Wakkach A, Guyon T, Bruand C, Tzartos S, Cohen-Kaminsky S, Berrih-Aknin S. Expression of acetylcholine receptor genes in human thymic epithelial cells: implications for myasthenia gravis. J Immunol 1996;157:3752-60.

[51] Schluep M, Willcox N, Vincent A, Dhoot GK, Newsom-Davis J. Acetylcholine receptors in human thymic myoid cells in situ: an immunohistological study. Ann Neurol 1987;22:212-22.

[52] Hill ME, Shiono H, Newsom-Davis J, Willcox N. The myasthenia gravis thymus: a rare source of human autoantibody-secreting plasma cells for testing potential therapeutics. J Neuroimmunol 2008;201-202:50-6.

[53] Melms A, Schalke BC, Kirchner T, Muller-Hermelink HK, Albert E, Wekerle H. Thymus in myasthenia gravis. Isolation of T-lymphocyte lines specific for the nicotinic acetylcholine receptor from thymuses of myasthenic patients. J Clin Invest 1988:81:902-8.

[54] Miyasaka M, Tanaka T. Lymphocyte trafficking across high endothelial venules: dogmas and enigmas. Nat Rev Immunol 2004;4:360-70.

[55] Germain RN, Robey EA, Cahalan MD. A decade of imaging cellular motility and interaction dynamics in the immune system. Science 2012:336:1676-81.

[56] Miller MJ, Wei SH, Parker I, Cahalan MD. Two-photon imaging of lymphocyte motility and antigen response in intact lymph node. Science 2002;296:186973.

[57] Stoll S, Delon J, Brotz TM, Germain RN. Dynamic imaging of T cell-dendritic cell interactions in lymph nodes. Science 2002;296:1873-6.

[58] Qi H, Egen JG, Huang AY, Germain RN. Extrafollicular activation of lymph node B cells by antigen-bearing dendritic cells. Science 2006;312:1672-6.

[59] Chtanova T, Han SJ, Schaeffer M, van Dooren GG, Herzmark P, Striepen B, et al. Dynamics of T cell, antigen-presenting cell, and pathogen interactions during recall responses in the lymph node. Immunity 2009;31:342-55.

[60] Bartholomaus I, Kawakami N, Odoardi F, Schlager C, Miljkovic D, Ellwart JW, et al. Effector T cell interactions with meningeal vascular structures in nascent autoimmune CNS lesions. Nature 2009;462:94-8.

[61] Lodygin D, Odoardi F, Schlager C, Korner H, Kitz A, Nosov M, et al A combination of fluorescent NFAT and H2B sensors uncovers dynamics of T cell activation in real time during CNS autoimmunity. Nat Med 2013;19:78490.

[62] Adjobimey T, Satoguina J, Oldenburg J, Hoerauf A, Layland LE. Co-activation through TLR4 and TLR9 but not TLR2 skews Treg-mediated modulation of Igs and induces IL-17 secretion in Treg:B cell co-cultures. Innate Immun 2014;20(1):12-23. [Epub 2013 Mar 25].

[63] Eisenbarth SC, Piggott DA, Huleatt JW, Visintin I, Herrick CA, Bottomly K Lipopolysaccharide-enhanced, toll-like receptor 4-dependent $\mathrm{T}$ helper cell type 2 responses to inhaled antigen. J Exp Med 2002;196:1645-51.

[64] Cavanagh LL, Weninger W. Dendritic cell behaviour in vivo: lessons learned from intravital two-photon microscopy. Immunol Cell Biol 2008;86:428-38.

[65] Pasare C, Medzhitov R. Toll pathway-dependent blockade of CD4+CD25+ cell-mediated suppression by dendritic cells. Science 2003;299:1033-6.

[66] Gomez-Martin D, Diaz-Zamudio M, Romo-Tena J, Ibarra-Sanchez MJ, AlcocerVarela J. Follicular helper T cells poise immune responses to the development of autoimmune pathology. Autoimmun Rev 2011:10:325-30.

[67] Luo C, Li Y, Liu W, Feng H, Wang H, Huang X, et al. Expansion of circulating counterparts of follicular helper T cells in patients with myasthenia gravis. J Neuroimmunol 2013;256:55-61.

[68] Xie X, Mu L, Yao X, Li N, Sun B, Li Y, et al. ATRA alters humoral responses associated with amelioration of EAMG symptoms by balancing Tfh/Tfr helper cell profiles. Clin Immunol 2013;148:162-76. 